MATHEMATICS OF COMPUTATION

Volume 79, Number 272, October 2010, Pages 2237-2264

S $0025-5718(10) 02329-\mathrm{X}$

Article electronically published on April 8, 2010

\title{
SPECTRAL METHOD ON QUADRILATERALS
}

\author{
GUO BEN-YU AND JIA HONG-LI
}

\begin{abstract}
In this paper, we investigate the spectral method on quadrilaterals. We introduce an orthogonal family of functions induced by Legendre polynomials, and establish some results on the corresponding orthogonal approximation. These results play important roles in the spectral method for partial differential equations defined on quadrilaterals. As examples of applications, we provide spectral schemes for two model problems and prove their spectral accuracy in Jacobi weighted Sobolev space. Numerical results coincide well with the analysis. We also investigate the spectral method on convex polygons whose solutions possess spectral accuracy. The approximation results of this paper are also applicable to other problems.
\end{abstract}

\section{INTRODUCTION}

During the past three decades, spectral method has gained increasing popularity in scientific computations; see [1]-7] and [9]-11] and the references therein. The standard spectral method is traditionally confined to periodic problems and problems defined on rectangular domains. However, many practical problems are set on complex domains. We usually use finite element methods for such problems. For obtaining accurate numerical results, it is also interesting to consider spectral methods and other high order methods for non-rectangular domains. Some authors proposed spectral methods for triangles, quadrilaterals and unbounded domains, see, e.g., [1], 2], 4], 14-17]. In particular, pseudospectral methods for polygons were developed with precise analysis; see [2, 5, 17] and the references therein.

In this paper, we investigate the spectral method on convex quadrilaterals. This work is motivated by several facts. For instance, we consider numerical solutions of partial differential equations defined on a polygon. In this case, we may divide the polygon into several convex quadrilaterals, and then use a spectral method on each quadrilateral. Next, for exterior problems of partial differential equations with a polygon obstacle, we could use mixed Laguerre-Legendre approximation outside a rectangle containing the obstacle, and use a spectral method for quadrilaterals on the remaining subdomain. Clearly, as the first step of those algorithms, we need to study spectral methods on quadrilaterals.

The paper is organized as follows. The next section is for preliminaries. In Section 3, we introduce an orthogonal system on a convex quadrilateral, induced by

Received by the editor July 15, 2008 and, in revised form, April 30, 2009 and June 21, 2009.

2010 Mathematics Subject Classification. Primary 65N35, 41A30, 35J05.

Key words and phrases. Orthogonal approximation on quadrilaterals, spectral method.

The work of this author is supported in part by NSF of China N.10871131, Science and Technology Commission of Shanghai Municipality, Grant N.075105118, Shanghai Leading Academic Discipline Project N.S30405, and Fund for E-institute of Shanghai Universities N.E03004.

(C)2010 American Mathematical Society Reverts to public domain 28 years from publication 


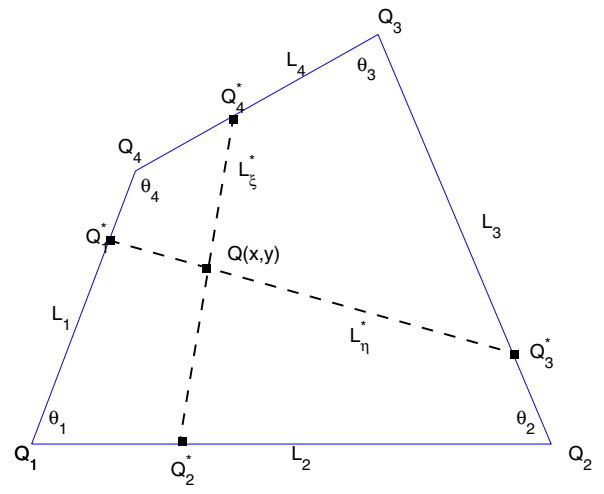

Figure 1. Quadrilateral $\Omega$.

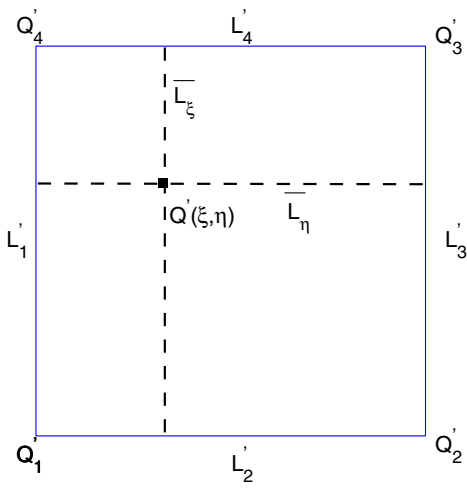

Figure 2. Square $S$.

the Legendre polynomials. Then we establish the basic results on the corresponding orthogonal approximation, which possesses spectral accuracy. Moreover, it still keeps high accuracy, even if the approximated function possesses certain singularities at the edges or the vertices of a quadrilateral. These results play important roles in the spectral method for partial differential equations defined on quadrilaterals. As examples of applications, we provide the spectral schemes for two model problems in Section 4, with the analysis of convergence. We describe their numerical implementation in Section 5, and present some numerical results in Section 6. In Section 7, we consider the spectral method for convex polygons and prove the spectral accuracy of their numerical solutions. The final section is for concluding remarks.

\section{Preliminaries}

Let $\Omega$ be a convex quadrilateral with the edges $L_{j}$, the vertices $Q_{j}=\left(x_{j}, y_{j}\right)$ and the angles $\theta_{j}, 1 \leq j \leq 4$; see Figure 1 . The length of $L_{j}$ is denoted by $l_{j}$. For any $Q=(x, y) \in \Omega$, we set

$$
\begin{aligned}
& \sigma_{1}(\xi, \eta)=\frac{1}{4}(1-\xi)(1-\eta), \\
& \sigma_{2}(\xi, \eta)=\frac{1}{4}(1+\xi)(1-\eta), \\
& \sigma_{3}(\xi, \eta)=\frac{1}{4}(1+\xi)(1+\eta), \\
& \sigma_{4}(\xi, \eta)=\frac{1}{4}(1-\xi)(1+\eta) .
\end{aligned}
$$

We make the variable transformation (cf. [2, 5, 17, 19]):

$$
x=x(\xi, \eta)=\sum_{j=1}^{4} x_{j} \sigma_{j}(\xi, \eta), \quad y=y(\xi, \eta)=\sum_{j=1}^{4} y_{j} \sigma_{j}(\xi, \eta) .
$$

More precisely,

$$
x=a_{0}+a_{1} \xi+a_{2} \eta+a_{3} \xi \eta, \quad y=b_{0}+b_{1} \xi+b_{2} \eta+b_{3} \xi \eta
$$

where

$$
\begin{array}{llrl}
a_{0}=\frac{1}{4}\left(x_{1}+x_{2}+x_{3}+x_{4}\right), & b_{0}=\frac{1}{4}\left(y_{1}+y_{2}+y_{3}+y_{4}\right), \\
a_{1}=\frac{1}{4}\left(-x_{1}+x_{2}+x_{3}-x_{4}\right), & b_{1}=\frac{1}{4}\left(-y_{1}+y_{2}+y_{3}-y_{4}\right), \\
a_{2}=\frac{1}{4}\left(-x_{1}-x_{2}+x_{3}+x_{4}\right), & b_{2}=\frac{1}{4}\left(-y_{1}-y_{2}+y_{3}+y_{4}\right), \\
a_{3}=\frac{1}{4}\left(x_{1}-x_{2}+x_{3}-x_{4}\right), & b_{3}=\frac{1}{4}\left(y_{1}-y_{2}+y_{3}-y_{4}\right) .
\end{array}
$$


By the transformation (2.2), the quadrilateral $\Omega$ is transformed to the reference square $S=\{(\xi, \eta) \mid-1<\xi, \eta<1\}$, with the edges $L_{j}^{\prime}$ and the vertices $Q_{j}^{\prime}=$ $\left(\xi_{j}, \eta_{j}\right), 1 \leq j \leq 4$; see Figure 2. In fact, $\xi_{1}=\xi_{4}=\eta_{1}=\eta_{2}=-1$ and $\xi_{2}=\xi_{3}=$ $\eta_{3}=\eta_{4}=1$.

It is noted that if $\Omega$ is a parallelogram, then $a_{3}=b_{3}=0$. In this case, the transformation (2.2) is an affine mapping. In particular, $a_{2}=a_{3}=b_{1}=b_{3}=0$ for any rectangle $\Omega$.

For simplicity, we denote $\frac{\partial x}{\partial \xi}$ by $\partial_{\xi} x$, etc. The Jacobi matrix of transformation $(2.2)$ is

$$
M_{\Omega}=\left(\begin{array}{ll}
\partial_{\xi} x & \partial_{\xi} y \\
\partial_{\eta} x & \partial_{\eta} y
\end{array}\right)=\left(\begin{array}{cc}
a_{1}+a_{3} \eta & b_{1}+b_{3} \eta \\
a_{2}+a_{3} \xi & b_{2}+b_{3} \xi
\end{array}\right) .
$$

Its Jacobian determinant is

$$
J_{\Omega}(\xi, \eta)=\left|\begin{array}{ll}
a_{1}+a_{3} \eta & b_{1}+b_{3} \eta \\
a_{2}+a_{3} \xi & b_{2}+b_{3} \xi
\end{array}\right|=d_{0}+d_{1} \xi+d_{2} \eta
$$

where

$$
d_{0}=a_{1} b_{2}-a_{2} b_{1}, \quad d_{1}=a_{1} b_{3}-a_{3} b_{1}, \quad d_{2}=a_{3} b_{2}-a_{2} b_{3} .
$$

By virtue of (2.4), we get

$$
\begin{aligned}
& d_{0}=\frac{1}{8}\left(x_{4}-x_{2}\right)\left(y_{1}-y_{3}\right)+\frac{1}{8}\left(x_{1}-x_{3}\right)\left(y_{2}-y_{4}\right), \\
& d_{1}=\frac{1}{8}\left(x_{4}-x_{3}\right)\left(y_{2}-y_{1}\right)+\frac{1}{8}\left(x_{2}-x_{1}\right)\left(y_{3}-y_{4}\right), \\
& d_{2}=\frac{1}{8}\left(x_{3}-x_{2}\right)\left(y_{4}-y_{1}\right)+\frac{1}{8}\left(x_{1}-x_{4}\right)\left(y_{3}-y_{2}\right) .
\end{aligned}
$$

Obviously, $J_{\Omega}(\xi, \eta)$ is a linear function of variables $\xi$ and $\eta$, and so reaches its extremum at the four vertices. A direct calculation shows that

$$
\begin{aligned}
& J_{\Omega}(-1,-1)=\frac{1}{4}\left(x_{4}-x_{2}\right) y_{1}+\frac{1}{4}\left(x_{1}-x_{4}\right) y_{2}+\frac{1}{4}\left(x_{2}-x_{1}\right) y_{4}, \\
& J_{\Omega}(1,-1)=\frac{1}{4}\left(x_{3}-x_{2}\right) y_{1}+\frac{1}{4}\left(x_{1}-x_{3}\right) y_{2}+\frac{1}{4}\left(x_{2}-x_{1}\right) y_{3}, \\
& J_{\Omega}(1,1)=\frac{1}{4}\left(x_{4}-x_{3}\right) y_{2}+\frac{1}{4}\left(x_{2}-x_{4}\right) y_{3}+\frac{1}{4}\left(x_{3}-x_{2}\right) y_{4}, \\
& J_{\Omega}(-1,1)=\frac{1}{4}\left(x_{4}-x_{3}\right) y_{1}+\frac{1}{4}\left(x_{1}-x_{4}\right) y_{3}+\frac{1}{4}\left(x_{3}-x_{1}\right) y_{4},
\end{aligned}
$$

or equivalently,

$$
\begin{aligned}
& J_{\Omega}(-1,-1)=\frac{1}{4}\left(x_{2}-x_{1}\right)\left(y_{4}-y_{1}\right)-\frac{1}{4}\left(x_{4}-x_{1}\right)\left(y_{2}-y_{1}\right), \\
& J_{\Omega}(1,-1)=\frac{1}{4}\left(x_{3}-x_{2}\right)\left(y_{1}-y_{2}\right)-\frac{1}{4}\left(x_{1}-x_{2}\right)\left(y_{3}-y_{2}\right), \\
& J_{\Omega}(1,1)=\frac{1}{4}\left(x_{4}-x_{3}\right)\left(y_{2}-y_{3}\right)-\frac{1}{4}\left(x_{2}-x_{3}\right)\left(y_{4}-y_{3}\right), \\
& J_{\Omega}(-1,1)=\frac{1}{4}\left(x_{1}-x_{4}\right)\left(y_{3}-y_{4}\right)-\frac{1}{4}\left(x_{3}-x_{4}\right)\left(y_{1}-y_{4}\right) .
\end{aligned}
$$

As pointed out by Strang and Fix in Section 3.3 of [18], we have

$$
\begin{aligned}
J_{\Omega}(-1,-1) & =l_{1} l_{2} \sin \theta_{1}, & & J_{\Omega}(1,-1)=l_{2} l_{3} \sin \theta_{2}, \\
J_{\Omega}(1,1) & =l_{3} l_{4} \sin \theta_{3}, & & J_{\Omega}(-1,1)=l_{4} l_{1} \sin \theta_{4} .
\end{aligned}
$$

Due to the convexity of $\Omega$, the above four quantities are positive. Thereby, there exist positive constants $\delta_{\Omega}$ and $\delta_{\Omega}^{*}$, such that

$$
\begin{aligned}
& 0<\delta_{\Omega}=\min \left\{J_{\Omega}(-1,-1), J_{\Omega}(1,-1), J_{\Omega}(1,1), J_{\Omega}(-1,1)\right\} \\
& \leq J_{\Omega}(\xi, \eta) \leq \max \left\{J_{\Omega}(-1,-1), J_{\Omega}(1,-1), J_{\Omega}(1,1), J_{\Omega}(-1,1)\right\}=\delta_{\Omega}^{*} .
\end{aligned}
$$

The inverse of transformation (2.2) is

$$
\xi=\xi(x, y), \quad \eta=\eta(x, y) .
$$


The explicit expressions of $\xi(x, y)$ and $\eta(x, y)$ are given in Appendix A of this paper. The Jacobi matrix of this inverse transformation is

$$
M_{S}=M_{\Omega}^{-1}=\left(\begin{array}{cc}
\partial_{x} \xi & \partial_{x} \eta \\
\partial_{y} \xi & \partial_{y} \eta
\end{array}\right)=\frac{1}{J_{\Omega}(\xi, \eta)}\left(\begin{array}{cc}
b_{2}+b_{3} \xi & -b_{1}-b_{3} \eta \\
-a_{2}-a_{3} \xi & a_{1}+a_{3} \eta
\end{array}\right)
$$

Thanks to (2.7), its Jacobian determinant $J_{S}(x, y)$ satisfies

$$
0<\frac{1}{\delta_{\Omega}^{*}} \leq J_{S}(x, y)=J_{\Omega}^{-1}(\xi, \eta) \leq \frac{1}{\delta_{\Omega}} .
$$

We now derive several relations, which will be used in forthcoming discussions. For any point $Q=(x, y) \in \Omega$, there exists the corresponding point $Q^{\prime}=(\xi(x, y)$, $\eta(x, y)) \in S$. Denote by $\bar{L}_{\eta}$ the line in $S$, which is parallel to the $\xi$-axis and passes by $Q^{\prime}$. Clearly, all points on this line have the same coordinate $\eta$. The mapping of the line $\bar{L}_{\eta}$, denoted by $L_{\eta}^{*}$, is not parallel to the $x$-axis usually. But it intersects the edges $L_{1}$ and $L_{3}$ at the points $Q_{1}^{*}=\left(x_{1}^{*}(x, y), y_{1}^{*}(x, y)\right)$ and $Q_{3}^{*}=\left(x_{3}^{*}(x, y), y_{3}^{*}(x, y)\right)$, respectively; see Figures 1 and 2. Similarly, all points on the line $\bar{L}_{\xi}$, which are parallel to the $\eta$-axis and pass by $Q^{\prime}$, have the same coordinate $\xi$. The mapping of the line $\bar{L}_{\xi}$, denoted by $L_{\xi}^{*}$, is not parallel to the $y$-axis usually; but it intersects the edges $L_{2}$ and $L_{4}$ at the points $Q_{2}^{*}=\left(x_{2}^{*}(x, y), y_{2}^{*}(x, y)\right)$ and $Q_{4}^{*}=\left(x_{4}^{*}(x, y), y_{4}^{*}(x, y)\right)$, respectively.

We now calculate $x_{j}^{*}(x, y)$ and $y_{j}^{*}(x, y), 1 \leq j \leq 4$. Indeed, (2.2) implies

$$
x_{1}^{*}=\sum_{j=1}^{4} x_{j} \sigma_{j}(-1, \eta), \quad y_{1}^{*}=\sum_{j=1}^{4} y_{j} \sigma_{j}(-1, \eta),
$$

whence

$$
x_{1}^{*}=\frac{1}{2}\left(x_{1}+x_{4}\right)+\frac{1}{2}\left(x_{4}-x_{1}\right) \eta, \quad y_{1}^{*}=\frac{1}{2}\left(y_{1}+y_{4}\right)+\frac{1}{2}\left(y_{4}-y_{1}\right) \eta .
$$

Similarly,

$$
\begin{aligned}
x_{2}^{*} & =\frac{1}{2}\left(x_{1}+x_{2}\right)+\frac{1}{2}\left(x_{2}-x_{1}\right) \xi, & y_{2}^{*} & =\frac{1}{2}\left(y_{1}+y_{2}\right)+\frac{1}{2}\left(y_{2}-y_{1}\right) \xi, \\
x_{3}^{*} & =\frac{1}{2}\left(x_{2}+x_{3}\right)+\frac{1}{2}\left(x_{3}-x_{2}\right) \eta, & y_{3}^{*} & =\frac{1}{2}\left(y_{2}+y_{3}\right)+\frac{1}{2}\left(y_{3}-y_{2}\right) \eta, \\
x_{4}^{*} & =\frac{1}{2}\left(x_{3}+x_{4}\right)+\frac{1}{2}\left(x_{3}-x_{4}\right) \xi, & y_{4}^{*} & =\frac{1}{2}\left(y_{3}+y_{4}\right)+\frac{1}{2}\left(y_{3}-y_{4}\right) \xi .
\end{aligned}
$$

Subtracting the first formula of (2.10) from the first formula of (2.2), we obtain

$$
x-x_{1}^{*}=\frac{1}{4}\left(x_{2}-x_{1}\right)(1+\xi)(1-\eta)+\frac{1}{4}\left(x_{3}-x_{4}\right)(1+\xi)(1+\eta) .
$$

Similarly,

$$
y-y_{1}^{*}=\frac{1}{4}\left(y_{2}-y_{1}\right)(1+\xi)(1-\eta)+\frac{1}{4}\left(y_{3}-y_{4}\right)(1+\xi)(1+\eta) .
$$

The above two equalities, together with (2.4), lead to

$$
x-x_{1}^{*}=(1+\xi)\left(a_{1}+a_{3} \eta\right), \quad y-y_{1}^{*}=(1+\xi)\left(b_{1}+b_{3} \eta\right) .
$$

Similarly,

$$
\begin{gathered}
x-x_{2}^{*}=(1+\eta)\left(a_{2}+a_{3} \xi\right), \quad y-y_{2}^{*}=(1+\eta)\left(b_{2}+b_{3} \xi\right), \\
x-x_{3}^{*}=-(1-\xi)\left(a_{1}+a_{3} \eta\right), \quad y-y_{3}^{*}=-(1-\xi)\left(b_{1}+b_{3} \eta\right), \\
x-x_{4}^{*}=-(1-\eta)\left(a_{2}+a_{3} \xi\right), \quad y-y_{4}^{*}=-(1-\eta)\left(b_{2}+b_{3} \xi\right) .
\end{gathered}
$$




\section{Orthogonal approximation on a QUADrilateral}

In this section, we establish the basic results on the orthogonal approximation on a convex quadrilateral.

3.1. Legendre orthogonal approximation. We first recall some recent results on the Legendre orthogonal approximation. Let $\Lambda_{\xi}=\{\xi|| \xi \mid<1\}$ and $\chi^{(\alpha, \beta)}(\xi)=$ $(1-\xi)^{\alpha}(1+\xi)^{\beta}, \alpha, \beta>-1$. We define the weighted space $L_{\chi^{(\alpha, \beta)}}^{2}\left(\Lambda_{\xi}\right)$ in the usual way, with the following inner product and norm,

$$
\begin{array}{r}
(u, v)_{\chi^{(\alpha, \beta)}, \Lambda_{\xi}}=\int_{\Lambda} u(\xi) v(\xi) \chi^{(\alpha, \beta)}(\xi) d \xi, \quad\|v\|_{\chi^{(\alpha, \beta)}, \Lambda_{\xi}}=(v, v)_{\chi^{(\alpha, \beta)}, \Lambda_{\xi}}^{\frac{1}{2}}, \\
\forall u, v \in L_{\chi^{(\alpha, \beta)}}^{2}\left(\Lambda_{\xi}\right) .
\end{array}
$$

We omit the subscript $\chi^{(\alpha, \beta)}$ in notation whenever $\alpha=\beta=0$.

The Legendre polynomial of degree $l$ is defined by

$$
L_{l}(\xi)=\frac{(-1)^{l}}{2^{l} l !} \partial_{\xi}^{l}\left(1-\xi^{2}\right)^{l} .
$$

The set of Legendre polynomials is a complete $L^{2}\left(\Lambda_{\xi}\right)$-orthogonal system. Moreover,

$$
\left\|L_{l}\right\|_{\Lambda_{\xi}}^{2}=\left(l+\frac{1}{2}\right)^{-1} .
$$

Let $N$ be any positive integer. Denote by $\mathcal{P}_{N}\left(\Lambda_{\xi}\right)$ the set of all polynomials of degree at most $N$. Moreover, $\mathcal{P}_{N}^{0}\left(\Lambda_{\xi}\right)=\mathcal{P}_{N}\left(\Lambda_{\xi}\right) \cap H_{0}^{1}\left(\Lambda_{\xi}\right)$. The $L^{2}\left(\Lambda_{\xi}\right)$-orthogonal projection $P_{N, \Lambda_{\xi}}: L^{2}\left(\Lambda_{\xi}\right) \rightarrow \mathcal{P}_{N}\left(\Lambda_{\xi}\right)$, is defined by

$$
\left(P_{N, \Lambda_{\xi}} v-v, \phi\right)_{\Lambda_{\xi}}=0, \quad \forall \phi \in \mathcal{P}_{N}\left(\Lambda_{\xi}\right)
$$

Throughout this paper, we denote by $c$ a generic positive constant independent of $N$ and any function. According to Theorem 2.1 of [12], we know that if $v \in$ $L^{2}\left(\Lambda_{\xi}\right), \partial_{\xi}^{r} v \in L_{\chi^{(r, r)}}^{2}\left(\Lambda_{\xi}\right)$, integers $r \geq 0$ and $r \leq N+1$, then

$$
\left\|P_{N, \Lambda} v-v\right\|_{\Lambda_{\xi}} \leq c N^{-r}\left\|\partial_{\xi}^{r} v\right\|_{\chi^{(r, r)}, \Lambda_{\xi}} .
$$

Next, the orthogonal projection $P_{N, \Lambda_{\xi}}^{1,0}: H_{0}^{1}\left(\Lambda_{\xi}\right) \rightarrow P_{N}^{0}\left(\Lambda_{\xi}\right)$, is defined by

$$
\left(\partial_{\xi}\left(P_{N, \Lambda_{\xi}}^{1,0} v-v\right), \partial_{\xi} \phi\right)_{\Lambda_{\xi}}=0, \quad \forall \phi \in \mathcal{P}_{N}^{0}\left(\Lambda_{\xi}\right)
$$

As a special case of Theorem 3.4 of [12], we have that if $v \in H_{0}^{1}\left(\Lambda_{\xi}\right), \partial_{\xi}^{r} v \in$ $L_{\chi(r-1, r-1)}^{2}\left(\Lambda_{\xi}\right)$ and integers $1 \leq r \leq N+1$, then

$$
\left\|\partial_{\xi}^{\mu}\left(P_{N, \Lambda_{\xi}}^{1,0} v-v\right)\right\|_{\Lambda_{\xi}} \leq c N^{\mu-r}\left\|\partial_{\xi}^{r} v\right\|_{\chi^{(r-1, r-1)}, \Lambda_{\xi}}, \quad \mu=0,1 .
$$

3.2. $L^{2}(\Omega)$-orthogonal approximation. We now consider the $L^{2}(\Omega)$-orthogonal approximation on the quadrilateral $\Omega$.

Let $\Lambda_{\eta}=\{\eta|| \eta \mid<1\}$. Clearly, the square $S=\Lambda_{\xi} \times \Lambda_{\eta}$. We denote the inner product and the norm of $L^{2}(\Omega)$ by $(u, v)_{\Omega}$ and $\|v\|_{\Omega}$, respectively.

We introduce the functions

$$
\begin{aligned}
\psi_{l, m}(x, y) & =L_{l}(\xi(x, y)) L_{m}(\eta(x, y)) J_{S}^{\frac{1}{2}}(x, y) \\
& =L_{l}(\xi(x, y)) L_{m}(\eta(x, y)) J_{\Omega}^{-\frac{1}{2}}(\xi(x, y), \eta(x, y)), \quad l, m \geq 0
\end{aligned}
$$


By virtue of (3.1), we get

$$
\begin{aligned}
\iint_{\Omega} \psi_{l, m}(x, y) \psi_{l^{\prime}, m^{\prime}}(x, y) d x d y & =\int_{\Lambda_{\xi}} L_{l}(\xi) L_{l^{\prime}}(\xi) d \xi \int_{\Lambda_{\eta}} L_{m}(\eta) L_{m^{\prime}}(\eta) d \eta \\
& =\left(l+\frac{1}{2}\right)^{-1}\left(m+\frac{1}{2}\right)^{-1} \delta_{l, l^{\prime}} \delta_{m, m^{\prime}},
\end{aligned}
$$

where $\delta_{l, l^{\prime}}$ is the Kronecker symbol. Moreover, the set of all $\psi_{l, m}$ is complete in the space $L^{2}(\Omega)$. Thus, for any $v \in L^{2}(\Omega)$, we have

$$
v(x, y)=\sum_{l=0}^{\infty} \sum_{m=0}^{\infty} \hat{v}_{l, m} \psi_{l, m}(x, y)
$$

with

$$
\hat{v}_{l, m}=\left(l+\frac{1}{2}\right)\left(m+\frac{1}{2}\right) \iint_{\Omega} v(x, y) \psi_{l, m}(x, y) d x d y .
$$

Furthermore, let

$$
V_{N}(\Omega)=\operatorname{span}\left\{\psi_{l, m}(x, y) \mid 0 \leq l, m \leq N\right\} .
$$

The $L^{2}(\Omega)$-orthogonal projection $P_{N}: L^{2}(\Omega) \rightarrow V_{N}(\Omega)$, is defined by

$$
\left(P_{N} v-v, \phi\right)_{\Omega}=0, \quad \forall \phi \in V_{N}(\Omega) .
$$

For description of approximation errors, we shall use the following notation:

$$
\begin{aligned}
A_{r, \Omega}(v) & =\sum_{k=0}^{r} \sum_{j=0}^{k} \delta_{\Omega}^{-r+k}\left(\left|d_{1}\right|^{r-k}\left\|\left(1-\xi^{2}\right)^{\frac{r}{2}}\left(a_{1}+a_{3} \eta\right)^{j}\left(b_{1}+b_{3} \eta\right)^{k-j} \partial_{x}^{j} \partial_{y}^{k-j} v\right\|_{\Omega}\right. \\
& \left.+\left|d_{2}\right|^{r-k}\left\|\left(1-\eta^{2}\right)^{\frac{r}{2}}\left(a_{2}+a_{3} \xi\right)^{j}\left(b_{2}+b_{3} \xi\right)^{k-j} \partial_{x}^{j} \partial_{y}^{k-j} v\right\|_{\Omega}\right) .
\end{aligned}
$$

Theorem 3.1. If $v \in L^{2}(\Omega), A_{r, \Omega}(v)$ is finite for integer $r \geq 0$, and $r \leq N+1$, then

$$
\left\|P_{N} v-v\right\|_{\Omega} \leq c N^{-r} A_{r, \Omega}(v) .
$$

Proof. By projection theorem,

$$
\left\|P_{N} v-v\right\|_{\Omega}^{2} \leq\|\phi-v\|_{\Omega}^{2}, \quad \forall \phi \in V_{N}(\Omega) .
$$

Let

$$
\begin{aligned}
& u(\xi, \eta)=v(x(\xi, \eta), y(\xi, \eta)) J_{\Omega}^{\frac{1}{2}}(\xi, \eta), \\
& \phi(x, y)=\left(P_{N, \Lambda_{\xi}} \bullet P_{N, \Lambda_{\eta}} u\right)(\xi(x, y), \eta(x, y)) J_{\Omega}^{-\frac{1}{2}}(\xi(x, y), \eta(x, y)) \in V_{N}(\Omega) .
\end{aligned}
$$

Due to $J_{S}(x, y)=J_{\Omega}^{-1}(\xi, \eta)$, we use (3.3) to obtain

$$
\begin{aligned}
\|v-\phi\|_{\Omega}^{2} & =\iint_{S}\left(u-P_{N, \Lambda_{\xi}} \bullet P_{N, \Lambda_{\eta}} u\right)^{2} d \xi d \eta \\
& \leq 2 \iint_{S}\left(u-P_{N, \Lambda_{\xi}} u\right)^{2} d \xi d \eta+2 \iint_{S}\left(P_{N, \Lambda_{\xi}} \bullet\left(u-P_{N, \Lambda_{\eta}} u\right)\right)^{2} d \xi d \eta \\
& \leq c N^{-2 r}\left\|\partial_{\xi}^{r} u\right\|_{L_{\chi}^{2(r, r)}}^{2}\left(\Lambda_{\xi}, L^{2}\left(\Lambda_{\eta}\right)\right) \\
& \leq c P^{-2 r}\left(\left\|\partial_{\xi}^{r} u\right\|_{L_{\chi}^{2}, \Lambda_{\eta}}^{2} u-u \|_{L^{2}\left(\Lambda_{\xi}, L^{2}\left(\Lambda_{\eta}\right)\right)}^{2}\left(\Lambda_{\xi}, L^{2}\left(\Lambda_{\eta}\right)\right)\right. \\
& \left.+\left\|\partial_{\eta}^{r} u\right\|_{L^{2}\left(\Lambda_{\xi}, L_{\chi}^{(r, r)}\right.}^{2}\left(\Lambda_{\eta}\right)\right)
\end{aligned}
$$

Thus, it remains to estimate the right side of (3.12). 
Let $\sigma(q)=(-1)^{q-1} 2^{-q}(2 q-3)$ !!. Thanks to (2.5), we have

$$
\partial_{\xi}^{q} J_{\Omega}^{\frac{1}{2}}=\sigma(q) d_{1}^{q} J_{\Omega}^{\frac{1}{2}-q}, \quad \partial_{\eta}^{q} J_{\Omega}^{\frac{1}{2}}=\sigma(q) d_{2}^{q} J_{\Omega}^{\frac{1}{2}-q} .
$$

Hence, we have from (3.11) that

$$
\begin{aligned}
& \partial_{\xi}^{r} u=\partial_{\xi}^{r}\left(v J_{\Omega}^{\frac{1}{2}}\right)=J_{\Omega}^{\frac{1}{2}} \sum_{k=0}^{r} C_{r}^{k} \sigma(r-k) d_{1}^{r-k} J_{\Omega}^{-r+k} \partial_{\xi}^{k} v, \\
& \partial_{\eta}^{r} u=\partial_{\eta}^{r}\left(v J_{\Omega}^{\frac{1}{2}}\right)=J_{\Omega}^{\frac{1}{2}} \sum_{k=0}^{r} C_{r}^{k} \sigma(r-k) d_{2}^{r-k} J_{\Omega}^{-r+k} \partial_{\eta}^{k} v .
\end{aligned}
$$

Furthermore, thanks to (2.3), we have

$$
\begin{gathered}
\partial_{\xi} v=\partial_{x} v \partial_{\xi} x+\partial_{y} v \partial_{\xi} y=\left(a_{1}+a_{3} \eta\right) \partial_{x} v+\left(b_{1}+b_{3} \eta\right) \partial_{y} v, \\
\partial_{\eta} v=\partial_{x} v \partial_{\eta} x+\partial_{y} v \partial_{\eta} y=\left(a_{2}+a_{3} \xi\right) \partial_{x} v+\left(b_{2}+b_{3} \xi\right) \partial_{y} v .
\end{gathered}
$$

Thus, we derive inductively that

$$
\begin{aligned}
& \partial_{\xi}^{k} v=\sum_{j=0}^{k} C_{k}^{j}\left(a_{1}+a_{3} \eta\right)^{j}\left(b_{1}+b_{3} \eta\right)^{k-j} \partial_{x}^{j} \partial_{y}^{k-j} v \\
& \partial_{\eta}^{k} v=\sum_{j=0}^{k} C_{k}^{j}\left(a_{2}+a_{3} \xi\right)^{j}\left(b_{2}+b_{3} \xi\right)^{k-j} \partial_{x}^{j} \partial_{y}^{k-j} v .
\end{aligned}
$$

Inserting (3.17) into (3.13) and inserting (3.18) into (3.14), respectively, we find that

$$
\partial_{\xi}^{r} u=J_{\Omega}^{\frac{1}{2}} \sum_{k=0}^{r} \sum_{j=0}^{k} C_{r}^{k} C_{k}^{j} \sigma(r-k) d_{1}^{r-k} J_{\Omega}^{-r+k}\left(a_{1}+a_{3} \eta\right)^{j}\left(b_{1}+b_{3} \eta\right)^{k-j} \partial_{x}^{j} \partial_{y}^{k-j} v
$$

$$
\partial_{\eta}^{r} u=J_{\Omega}^{\frac{1}{2}} \sum_{k=0}^{r} \sum_{j=0}^{k} C_{r}^{k} C_{k}^{j} \sigma(r-k) d_{2}^{r-k} J_{\Omega}^{-r+k}\left(a_{2}+a_{3} \xi\right)^{j}\left(b_{2}+b_{3} \xi\right)^{k-j} \partial_{x}^{j} \partial_{y}^{k-j} v .
$$

Therefore,

$$
\begin{aligned}
& \left\|\partial_{\xi}^{r} u\right\|_{L_{\chi^{(r, r)}\left(\Lambda_{\xi}, L^{2}\left(\Lambda_{\eta}\right)\right)}} \\
& \quad \leq c \sum_{k=0}^{r} \sum_{j=0}^{k}\left|d_{1}\right|^{r-k} \delta_{\Omega}^{-r+k}\left\|\left(1-\xi^{2}\right)^{\frac{r}{2}}\left(a_{1}+a_{3} \eta\right)^{j}\left(b_{1}+b_{3} \eta\right)^{k-j} \partial_{x}^{j} \partial_{y}^{k-j} v\right\|_{\Omega} .
\end{aligned}
$$

Similarly,

$$
\begin{aligned}
& \left\|\partial_{\eta}^{r} u\right\|_{L^{2}\left(\Lambda_{\xi}, L^{2} \chi^{(r, r)}\left(\Lambda_{\eta}\right)\right)} \\
& \quad \leq c \sum_{k=0}^{r} \sum_{j=0}^{k}\left|d_{2}\right|^{r-k} \delta_{\Omega}^{-r+k}\left\|\left(1-\eta^{2}\right)^{\frac{r}{2}}\left(a_{2}+a_{3} \xi\right)^{j}\left(b_{2}+b_{3} \xi\right)^{k-j} \partial_{x}^{j} \partial_{y}^{k-j} v\right\|_{\Omega} .
\end{aligned}
$$

Finally, the desired result follows from a combination of (3.12), (3.21) and (3.22). 
Remark 3.1. By virtue of (2.15)-(2.18),

$$
\begin{array}{ll}
a_{1}+a_{3} \eta=\frac{x-x_{1}^{*}}{1+\xi}=-\frac{x-x_{3}^{*}}{1-\xi}, & b_{1}+b_{3} \eta=\frac{y-y_{1}^{*}}{1+\xi}=-\frac{y-y_{3}^{*}}{1-\xi}, \\
a_{2}+a_{3} \xi=\frac{x-x_{2}^{*}}{1+\eta}=-\frac{x-x_{4}^{*}}{1-\eta}, & b_{2}+b_{3} \xi=\frac{y-y_{2}^{*}}{1+\eta}=-\frac{y-y_{4}^{*}}{1-\eta} .
\end{array}
$$

Consequently,

$$
\begin{array}{ll}
\left|a_{1}+a_{3} \eta\right|=\sqrt{-\frac{\left(x-x_{1}^{*}\right)\left(x-x_{3}^{*}\right)}{1-\xi^{2}}}, & \left|b_{1}+b_{3} \eta\right|=\sqrt{-\frac{\left(y-y_{1}^{*}\right)\left(y-y_{3}^{*}\right)}{1-\xi^{2}}}, \\
\left|a_{2}+a_{3} \xi\right|=\sqrt{-\frac{\left(x-x_{2}^{*}\right)\left(x-x_{4}^{*}\right)}{1-\eta^{2}}}, & \left|b_{2}+b_{3} \xi\right|=\sqrt{-\frac{\left(y-y_{2}^{*}\right)\left(y-y_{4}^{*}\right)}{1-\eta^{2}}} .
\end{array}
$$

Therefore, (3.10) implies

$$
\left\|P_{N} v-v\right\|_{\Omega} \leq c N^{-r} A_{r, \Omega}^{*}(v)
$$

where

$$
\begin{aligned}
A_{r, \Omega}^{*}(v) & =\sum_{k=0}^{r} \sum_{j=0}^{k} \delta_{\Omega}^{-r+k}\left(\left|d_{1}\right|^{r-k}\left\|\left(\left(x-x_{1}^{*}\right)\left(x-x_{3}^{*}\right)\right)^{\frac{j}{2}}\left(\left(y-y_{1}^{*}\right)\left(y-y_{3}^{*}\right)\right)^{\frac{k-j}{2}} \partial_{x}^{j} \partial_{y}^{k-j} v\right\|_{\Omega}\right. \\
& \left.+\left|d_{2}\right|^{r-k}\left\|\left(\left(x-x_{2}^{*}\right)\left(x-x_{4}^{*}\right)\right)^{\frac{j}{2}}\left(\left(y-y_{2}^{*}\right)\left(y-y_{4}^{*}\right)\right)^{\frac{k-j}{2}} \partial_{x}^{j} \partial_{y}^{k-j} v\right\|_{\Omega}\right) .
\end{aligned}
$$

On the other hand, let $x_{5}=x_{1}, y_{5}=y_{1}$, and

$$
\sigma_{\Omega}=\max _{(\xi, \eta) \in S}\left(\left|b_{2}+b_{3} \xi\right|,\left|b_{1}+b_{3} \eta\right|,\left|a_{2}+a_{3} \xi\right|,\left|a_{1}+a_{3} \eta\right|\right) .
$$

It can be checked that

$$
\sigma_{\Omega}=\frac{1}{2} \max _{1 \leq j \leq 4}\left(\left|x_{j}-x_{j+1}\right|,\left|y_{j}-y_{j+1}\right|\right) .
$$

Accordingly, by (3.10),

$$
\left\|P_{N} v-v\right\|_{\Omega} \leq c N^{-r} A_{r, \Omega}^{* *}(v)
$$

where

$A_{r, \Omega}^{* *}(v)=\sum_{k=0}^{r} \sum_{j=0}^{k} \delta_{\Omega}^{-r+k} \sigma_{\Omega}^{k}\left(\left|d_{1}\right|^{r-k}||\left(1-\xi^{2}\right)^{\frac{r}{2}} \partial_{x}^{j} \partial_{y}^{k-j} v\left\|_{\Omega}+\left|d_{2}\right|^{r-k}\right\|\left(1-\eta^{2}\right)^{\frac{r}{2}} \partial_{x}^{j} \partial_{y}^{k-j} v \|_{\Omega}\right)$.

Remark 3.2. We see from the definition of $A_{r, \Omega}(v)$ and (3.24) that in the norm of derivative $\partial_{x}^{j} \partial_{y}^{k-j} v$, there are the weight functions

$$
\begin{aligned}
& f_{j, k}=\left|\left(1-\xi^{2}\right)^{r-k}\left(\left(x-x_{1}^{*}\right)\left(x-x_{3}^{*}\right)\right)^{j}\left(\left(y-y_{1}^{*}\right)\left(y-y_{3}^{*}\right)\right)^{k-j}\right|, \quad 0 \leq j \leq k \leq r, \\
& g_{j, k}=\left|\left(1-\eta^{2}\right)^{r-k}\left(\left(x-x_{2}^{*}\right)\left(x-x_{4}^{*}\right)\right)^{j}\left(\left(y-y_{2}^{*}\right)\left(y-y_{4}^{*}\right)\right)^{k-j}\right|, 0 \leq j \leq k \leq r .
\end{aligned}
$$

Let $\left|Q-Q_{\nu}^{*}\right|$ be the distance between $Q(x, y)$ and $Q_{\nu}^{*}(x, y)$. Clearly,

$$
\begin{array}{ll}
f_{j, k} \leq c\left(1-\xi^{2}\right)^{r-k}\left|Q-Q_{\nu}^{*}\right|^{2 k}, & 0 \leq k \leq r, \nu=1,3, \\
g_{j, k} \leq c\left(1-\eta^{2}\right)^{r-k}\left|Q-Q_{\nu}^{*}\right|^{2 k}, & 0 \leq k \leq r, \nu=2,4 .
\end{array}
$$

Thus, all $f_{j, k}$ tend to zero as $Q(x, y)$ goes to $L_{1}$ or $L_{3}$, while all $g_{j, k}$ tend to zero as $Q(x, y)$ goes to $L_{2}$ or $L_{4}$. Moreover, the higher the order $k$ of derivative, the smaller the weight functions $f_{j, k}$ and $g_{j, k}$. Next, let $Q_{5}(x, y)=Q_{1}(x, y)$. We find, especially from (2.11) - 2.14), that the points $Q_{\nu}^{*}(x, y)$ and $Q_{\nu+1}^{*}(x, y)$ tend to the same vertex $Q_{\nu}$, as the point $Q(x, y)$ goes to $Q_{\nu}, 1 \leq \nu \leq 4$. Therefore, the corresponding weight functions tend to zero simultaneously. As a result, $\left\|P_{N} v-v\right\|_{\Omega}$ still keeps the order $N^{-r}$, even if the approximated function has the singularity 
at the vertices, such as $\left|\partial_{x}^{j} \partial_{y}^{k-j} v\right|=\mathrm{o}\left(\left(1-\xi^{2}\right)^{\frac{-r+k}{2}}\left|Q-Q_{\nu}^{*}\right|^{\frac{-2 k-1}{2}}\right), \nu=1,3$ and $\left.\left|\partial_{x}^{j} \partial_{y}^{k-j} v\right|=\mathrm{o}\left(\left(1-\eta^{2}\right)^{\frac{-r+k}{2}}\left|Q-Q_{\nu}^{*}\right|\right)^{\frac{-2 k-1}{2}}\right), \nu=2,4$.

Remark 3.3. If $\Omega=\{(x, y)|| x|<a| y \mid,<b, a, b,>0\}$, then $a_{1}=a, b_{2}=b, a_{2}=$ $a_{3}=b_{1}=b_{3}=0$. In this case, $\partial_{\xi} v=a \partial_{x} v, \partial_{\eta} v=b \partial_{y} v, J_{\Omega}=a b, x_{1}^{*}(x, y)=$ $-a, x_{2}^{*}(x, y)=x_{4}^{*}(x, y)=x, x_{3}^{*}(x, y)=a, y_{1}^{*}(x, y)=y_{3}^{*}(x, y)=y, y_{2}^{*}(x, y)=$ $-b, y_{4}^{*}(x, y)=b$, and $\xi(x, y)=\frac{x}{a}, \eta(x, y)=\frac{y}{b}$. Therefore,

$$
\left\|P_{N} v-v\right\|_{\Omega} \leq c N^{-r}\left(\left\|\left(a^{2}-x^{2}\right)^{\frac{r}{2}} \partial_{x}^{r} v\right\|_{\Omega}+\left\|\left(b^{2}-y^{2}\right)^{\frac{r}{2}} \partial_{y}^{r} v\right\|_{\Omega}\right) .
$$

It is noted that in this special case, the $L^{2}(\Omega)$-orthogonal approximation keeps the same spectral accuracy, even if the considered function possesses certain singularities at the edges of the quadrilateral. If, in addition, $a=b=1$, then the above estimate turns out to be the same result as in [13.

3.3. $H_{0}^{1}(\Omega)$-orthogonal approximation. We now turn to the $H_{0}^{1}(\Omega)$-orthogonal approximation. According to the Poincaré inequality, there exists a positive constant $c_{\Omega}$ such that

$$
\|w\|_{\Omega} \leq c_{\Omega}\|\nabla w\|_{\Omega}, \quad \forall w \in H_{0}^{1}(\Omega) .
$$

For simplicity of statements, let $\left(a_{1}+a_{3} \eta\right)_{\dagger}^{\nu}=\left(a_{1}+a_{3} \eta\right)^{\nu}$ for $\nu \geq 0$, and $\left(a_{1}+\right.$ $\left.a_{3} \eta\right)_{\dagger}^{\nu}=0$ otherwise. The meanings of $\left(b_{1}+b_{3} \eta\right)_{\dagger}^{\nu},\left(a_{2}+a_{3} \xi\right)_{\dagger}^{\nu}$ and $\left(b_{2}+b_{3} \xi\right)_{\dagger}^{\nu}$ are similar. Let $\gamma_{\Omega}=\max \left(\left|a_{3}\right|,\left|b_{3}\right|\right)$. Clearly, $\gamma_{\Omega} \leq \sigma_{\Omega}$. In order to describe the approximation error, we also introduce the following notation:

$$
\begin{aligned}
& B_{r, \Omega}(v)=\sigma_{\Omega}\left(\left(\left|d_{1}\right|+\left|d_{2}\right|\right) \delta_{\Omega}^{-1}+1\right) \\
& \cdot\left(\sum _ { k = 0 } ^ { r } \sum _ { j = 0 } ^ { k } \delta _ { \Omega } ^ { - r + k - 1 } \left\{\left|d_{1}\right|^{r-k}\left\|\left(1-\xi^{2}\right)^{\frac{r-1}{2}}\left(a_{1}+a_{3} \eta\right)^{j}\left(b_{1}+b_{3} \eta\right)^{k-j} \partial_{x}^{j} \partial_{y}^{k-j} v\right\|_{\Omega}\right.\right. \\
& \left.+\left|d_{2}\right|^{r-k}\left\|\left(1-\eta^{2}\right)^{\frac{r-1}{2}}\left(a_{2}+a_{3} \xi\right)^{j}\left(b_{2}+b_{3} \xi\right)^{k-j} \partial_{x}^{j} \partial_{y}^{k-j} v\right\|_{\Omega}\right\} \\
& +\sum_{k=0}^{r-1} \sum_{j=0}^{k} \delta_{\Omega}^{-r+k}\left\{| d _ { 1 } | ^ { r - k - 1 } \left[\left|d_{2}\right| \delta_{\Omega}^{-1}\left\|\left(1-\xi^{2}\right)^{\frac{r-2}{2}}\left(a_{1}+a_{3} \eta\right)^{j}\left(b_{1}+b_{3} \eta\right)^{k-j} \partial_{x}^{j} \partial_{y}^{k-j} v\right\|_{\Omega}\right.\right. \\
& +\gamma_{\Omega}\left\|\left(1-\xi^{2}\right)^{\frac{r-2}{2}}\left(a_{1}+a_{3} \eta\right)_{\dagger}^{j-1}\left(b_{1}+b_{3} \eta\right)^{k-j} \partial_{x}^{j} \partial_{y}^{k-j} v\right\|_{\Omega} \\
& +\gamma_{\Omega}\left\|\left(1-\xi^{2}\right)^{\frac{r-2}{2}}\left(a_{1}+a_{3} \eta\right)^{j}\left(b_{1}+b_{3} \eta\right)_{\dagger}^{k-j-1} \partial_{x}^{j} \partial_{y}^{k-j} v\right\|_{\Omega} \\
& +\sigma_{\Omega}\left\|\left(1-\xi^{2}\right)^{\frac{r-2}{2}}\left(a_{1}+a_{3} \eta\right)^{j}\left(b_{1}+b_{3} \eta\right)^{k-j} \partial_{x}^{j+1} \partial_{y}^{k-j} v\right\|_{\Omega} \\
& \left.+\sigma_{\Omega}\left\|\left(1-\xi^{2}\right)^{\frac{r-2}{2}}\left(a_{1}+a_{3} \eta\right)^{j}\left(b_{1}+b_{3} \eta\right)^{k-j} \partial_{x}^{j} \partial_{y}^{k-j+1} v\right\|_{\Omega}\right] \\
& +\left|d_{2}\right|^{r-k-1}\left[\left|d_{1}\right| \delta_{\Omega}^{-1}||\left(1-\eta^{2}\right)^{\frac{r-2}{2}}\left(a_{2}+a_{3} \xi\right)^{j}\left(b_{2}+b_{3} \xi\right)^{k-j} \partial_{x}^{j} \partial_{y}^{k-j} v \|_{\Omega}\right. \\
& +\gamma_{\Omega}\left\|\left(1-\eta^{2}\right)^{\frac{r-2}{2}}\left(a_{2}+a_{3} \xi\right)_{\dagger}^{j-1}\left(b_{2}+b_{3} \xi\right)^{k-j} \partial_{x}^{j} \partial_{y}^{k-j} v\right\|_{\Omega} \\
& +\gamma_{\Omega}\left\|\left(1-\eta^{2}\right)^{\frac{r-2}{2}}\left(a_{2}+a_{3} \xi\right)^{j}\left(b_{2}+b_{3} \xi\right)_{\dagger}^{k-j-1} \partial_{x}^{j} \partial_{y}^{k-j} v\right\|_{\Omega} \\
& +\sigma_{\Omega}\left\|\left(1-\eta^{2}\right)^{\frac{r-2}{2}}\left(a_{2}+a_{3} \xi\right)^{j}\left(b_{2}+b_{3} \xi\right)^{k-j} \partial_{x}^{j+1} \partial_{y}^{k-j} v\right\|_{\Omega} \\
& \left.\left.\left.+\sigma_{\Omega}\left\|\left(1-\eta^{2}\right)^{\frac{r-2}{2}}\left(a_{2}+a_{3} \xi\right)^{j}\left(b_{2}+b_{3} \xi\right)^{k-j} \partial_{x}^{j} \partial_{y}^{k-j+1} v\right\|_{\Omega}\right]\right\}\right) .
\end{aligned}
$$

Let $V_{N}^{0}(\Omega)=H_{0}^{1}(\Omega) \bigcap V_{N}(\Omega)$. The orthogonal projection $P_{N}^{1,0}: H_{0}^{1}(\Omega) \rightarrow V_{N}^{0}(\Omega)$ is defined by

$$
\left(\nabla\left(P_{N}^{1,0} v-v\right), \nabla \phi\right)_{\Omega}=0, \forall \phi \in V_{N}^{0}(\Omega)
$$


Theorem 3.2. If $v \in H_{0}^{1}(\Omega)$ and $B_{r, \Omega}(v)$ is finite for integers $2 \leq r \leq N+1$, then

$$
\left\|\nabla\left(P_{N}^{1,0} v-v\right)\right\|_{\Omega} \leq c N^{1-r} B_{r, \Omega}(v), \quad\left\|P_{N}^{1,0} v-v\right\|_{\Omega} \leq c c_{\Omega}^{*} N^{-r} B_{r, \Omega}(v),
$$

where $c_{\Omega}^{*}$ is a positive constant depending on $\Omega$.

Proof. By the projection theorem,

$$
\left\|\nabla\left(P_{N}^{1,0} v-v\right)\right\|_{\Omega} \leq\|\nabla(\phi-v)\|_{\Omega}, \quad \forall \phi \in V_{N}^{0}(\Omega) .
$$

Let $u(\xi, \eta)$ be the same as in (3.11), and $\psi(\xi, \eta)=\left(P_{N, \Lambda_{\xi}}^{1,0} \bullet P_{N, \Lambda_{\eta}}^{1,0} u\right)(\xi, \eta)$. Take

$$
\phi(x, y)=\psi(\xi(x, y), \eta(x, y)) J_{\Omega}^{-\frac{1}{2}}(\xi(x, y), \eta(x, y)) \in V_{N}^{0}(\Omega) .
$$

We denote by $\|w\|_{S}$ the norm of the space $L^{2}(S)$, and $\nabla_{S} w=\left(\partial_{\xi} w, \partial_{\eta} w\right)^{T}$. It can be shown that $\nabla_{S} w=M_{\Omega} \nabla w$. Thus by (2.8),,$\nabla w=M_{S} \nabla_{S} w$. Hence, we use (2.8) and (2.5) successively to obtain

$$
\begin{aligned}
\partial_{x}(\phi-v) & =\left(b_{2}+b_{3} \xi\right) J_{\Omega}^{-1} \partial_{\xi}\left((\psi-u) J_{\Omega}^{-\frac{1}{2}}\right)-\left(b_{1}+b_{3} \eta\right) J_{\Omega}^{-1} \partial_{\eta}\left((\psi-u) J_{\Omega}^{-\frac{1}{2}}\right) \\
& =\left(b_{2}+b_{3} \xi\right) J_{\Omega}^{-\frac{3}{2}}\left(\partial_{\xi}(\psi-u)-\frac{1}{2} d_{1} J_{\Omega}^{-1}(\psi-u)\right) \\
& -\left(b_{1}+b_{3} \eta\right) J_{\Omega}^{-\frac{3}{2}}\left(\partial_{\eta}(\psi-u)-\frac{1}{2} d_{2} J_{\Omega}^{-1}(\psi-u)\right) .
\end{aligned}
$$

With the aid of (2.9), (3.26) and the Poincaré inequality on $S$, a direct calculation gives

$$
\begin{aligned}
\left\|\partial_{x}(\phi-v)\right\|_{\Omega} & \leq c \sigma_{\Omega} \delta_{\Omega}^{-1}\left(\|\nabla(\psi-u)\|_{S}+\left(\left|d_{1}\right|+\left|d_{2}\right|\right) \delta_{\Omega}^{-1}\|\psi-u\|_{S}\right) \\
& \leq c \sigma_{\Omega} \delta_{\Omega}^{-1}\left(\left(\left|d_{1}\right|+\left|d_{2}\right|\right) \delta_{\Omega}^{-1}+1\right)\left\|\nabla_{S}(\psi-u)\right\|_{S}
\end{aligned}
$$

We can estimate $\left\|\partial_{y}(\phi-v)\right\|_{\Omega}$ in the same manner. Accordingly,

$$
\|\nabla(\phi-v)\|_{\Omega} \leq c \sigma_{\Omega} \delta_{\Omega}^{-1}\left(\left(\left|d_{1}\right|+\left|d_{2}\right|\right) \delta_{\Omega}^{-1}+1\right)\left\|\nabla_{S}(\psi-u)\right\|_{S} .
$$

We now estimate $\left\|\nabla_{S}(\psi-u)\right\|_{S}$. Clearly,

$$
\begin{aligned}
\left\|\partial_{\xi}(\psi-u)\right\|_{S}^{2} & =\left\|\partial_{\xi}\left(P_{N, \Lambda_{\xi}}^{1,0} \bullet P_{N, \Lambda_{\eta}}^{1,0} u-u\right)\right\|_{S}^{2} \\
& \leq\left\|\partial_{\xi}\left(P_{N, \Lambda_{\xi}}^{1,0} u-u\right)\right\|_{S}^{2}+\left\|\partial_{\xi} P_{N, \Lambda_{\xi}}^{1,0}\left(P_{N, \Lambda_{\eta}}^{1,0} u-u\right)\right\|_{S}^{2} .
\end{aligned}
$$

Using (3.5) with $\mu=1$ gives

$$
\left\|\partial_{\xi}\left(P_{N, \Lambda_{\xi}}^{1,0} u-u\right)\right\|_{S}^{2} \leq c N^{2-2 r}\left\|\partial_{\xi}^{r} u\right\|_{L^{(r-1, r-1)}}^{2}\left(\Lambda_{\xi}, L^{2}\left(\Lambda_{\eta}\right)\right) .
$$

Using (3.5) with $r=\mu=1$ gives

$$
\left\|\partial_{\xi} P_{N, \Lambda_{\xi}}^{1,0}\left(P_{N, \Lambda_{\eta}}^{1,0} u-u\right)\right\|_{S}^{2} \leq\left\|\partial_{\xi}\left(P_{N, \Lambda_{\eta}}^{1,0} u-u\right)\right\|_{L^{2}\left(\Lambda_{\xi}, L^{2}\left(\Lambda_{\eta}\right)\right)}^{2} .
$$

Thereby, using (3.5) with $\mu=0$ again yields

$$
\left.\left\|\partial_{\xi} P_{N, \Lambda_{\xi}}^{1,0}\left(P_{N, \Lambda_{\eta}}^{1,0} u-u\right)\right\|_{S}^{2} \leq c N^{2-2 r}\left\|\partial_{\xi} \partial_{\eta}^{r-1} u\right\|_{L^{2}\left(\Lambda_{\xi}, L_{\chi}^{2}\right.}^{2(r-2, r-2)}\left(\Lambda_{\eta}\right)\right) .
$$

We can estimate $\left\|\partial_{\eta}(\psi-u)\right\|_{S}^{2}$ similarly. Consequently, we get that

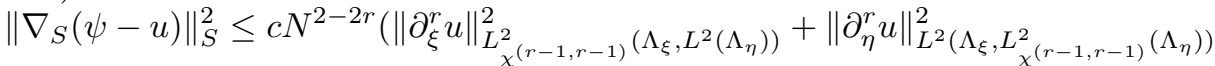

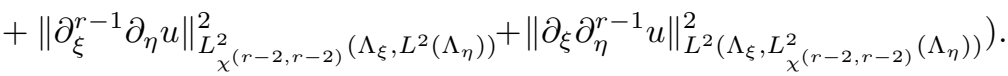


We next estimate the right side of (3.33). By (3.19), (3.20) and the same argument as in the derivations of (3.21) and (3.22), we verify that

$$
\begin{aligned}
& \left\|\partial_{\xi}^{r} u\right\|_{L_{\chi^{(r-1, r-1)}\left(\Lambda_{\xi}, L^{2}\left(\Lambda_{\eta}\right)\right)}^{2}} \\
& \quad \leq c \sum_{k=0}^{r} \sum_{j=0}^{k}\left|d_{1}\right|^{r-k} \delta_{\Omega}^{-r+k}\left\|\left(1-\xi^{2}\right)^{\frac{r-1}{2}}\left(a_{1}+a_{3} \eta\right)^{j}\left(b_{1}+b_{3} \eta\right)^{k-j} \partial_{x}^{j} \partial_{y}^{k-j} v\right\|_{\Omega}, \\
& \left.\left\|\partial_{\eta}^{r} u\right\|_{L^{2}\left(\Lambda_{\xi}, L^{2}\right.}^{\chi^{(r-1, r-1)}}\left(\Lambda_{\eta}\right)\right) \\
& \quad \leq c \sum_{k=0}^{r} \sum_{j=0}^{k}\left|d_{2}\right|^{r-k} \delta_{\Omega}^{-r+k}\left\|\left(1-\eta^{2}\right)^{\frac{r-1}{2}}\left(a_{2}+a_{3} \xi\right)^{j}\left(b_{2}+b_{3} \xi\right)^{k-j} \partial_{x}^{j} \partial_{y}^{k-j} v\right\|_{\Omega} .
\end{aligned}
$$

Furthermore, thanks to (2.3) and (2.5), we have $\partial_{\xi} J_{\Omega}=d_{1}$ and $\partial_{\eta} J_{\Omega}=d_{2}$. Moreover,

$$
\begin{aligned}
& \partial_{\xi}\left(\partial_{x}^{j} \partial_{y}^{k-j} v\right)=\left(a_{1}+a_{3} \eta\right) \partial_{x}^{j+1} \partial_{y}^{k-j} v+\left(b_{1}+b_{3} \eta\right) \partial_{x}^{j} \partial_{y}^{k-j+1} v, \\
& \partial_{\eta}\left(\partial_{x}^{j} \partial_{y}^{k-j} v\right)=\left(a_{2}+a_{3} \xi\right) \partial_{x}^{j+1} \partial_{y}^{k-j} v+\left(b_{2}+b_{3} \xi\right) \partial_{x}^{j} \partial_{y}^{k-j+1} v .
\end{aligned}
$$

Therefore, by differentiating (3.19) with respect to $\eta$, we obtain

$$
\begin{aligned}
\partial_{\xi}^{r-1} \partial_{\eta} u & =\sum_{k=0}^{r-1} \sum_{j=0}^{k} C_{r-1}^{k} C_{k}^{j} \sigma(r-k-1) d_{1}^{r-k-1} J_{\Omega}^{-r+k+\frac{1}{2}} \\
& \cdot\left[\left(d_{2}\left(-r+k+\frac{3}{2}\right)\left(a_{1}+a_{3} \eta\right)^{j}\left(b_{1}+b_{3} \eta\right)^{k-j}\right.\right. \\
& +J_{\Omega}\left(a_{3}\left(a_{1}+a_{3} \eta\right)_{\dagger}^{j-1}\left(b_{1}+b_{3} \eta\right)^{k-j}+b_{3}\left(a_{1}+a_{3} \eta\right)^{j}\left(b_{1}+b_{3} \eta\right)_{\dagger}^{k-j-1}\right) \partial_{x}^{j} \partial_{y}^{k-j} v \\
& \left.+J_{\Omega}\left(a_{1}+a_{3} \eta\right)^{j}\left(b_{1}+b_{3} \eta\right)^{k-j}\left(\left(a_{2}+a_{3} \xi\right) \partial_{x}^{j+1} \partial_{y}^{k-j} v+\left(b_{2}+b_{3} \xi\right) \partial_{x}^{j} \partial_{y}^{k-j+1} v\right)\right] .
\end{aligned}
$$

Similarly, by differentiating (3.20) with respect to $\xi$, we obtain

$$
\begin{aligned}
& \partial_{\xi} \partial_{\eta}^{r-1} u=\sum_{k=0}^{r-1} \sum_{j=0}^{k} C_{r-1}^{k} C_{k}^{j} \sigma(r-k-1) d_{2}^{r-k-1} J_{\Omega}^{-r+k+\frac{1}{2}} \\
& \quad \cdot\left[\left(d_{1}\left(-r+k+\frac{3}{2}\right)\left(a_{2}+a_{3} \xi\right)^{j}\left(b_{2}+b_{3} \xi\right)^{k-j}\right.\right. \\
& \quad+J_{\Omega}\left(a_{3}\left(a_{2}+a_{3} \xi\right)_{\dagger}^{j-1}\left(b_{2}+b_{3} \xi\right)^{k-j}+b_{3}\left(a_{2}+a_{3} \xi\right)^{j}\left(b_{2}+b_{3} \xi\right)_{\dagger}^{k-j-1}\right) \partial_{x}^{j} \partial_{y}^{k-j} v \\
& \left.\quad+J_{\Omega}\left(a_{2}+a_{3} \xi\right)^{j}\left(b_{2}+b_{3} \xi\right)^{k-j}\left(\left(a_{1}+a_{3} \eta\right) \partial_{x}^{j+1} \partial_{y}^{k-j} v+\left(b_{1}+b_{3} \eta\right) \partial_{x}^{j} \partial_{y}^{k-j+1} v\right)\right] .
\end{aligned}
$$

Then, following the same line as in the derivations of (3.21) and (3.22), we obtain

$$
\begin{aligned}
\left\|\partial_{\xi}^{r-1} \partial_{\eta} u\right\|_{L_{\chi^{(r-2, r-2)}}^{2}\left(\Lambda_{\xi}, L^{2}\left(\Lambda_{\eta}\right)\right)} \leq c \sum_{k=0}^{r-1} \sum_{j=0}^{k}\left|d_{1}\right|^{r-k-1} \delta_{\Omega}^{-r+k+1} \\
\quad \cdot\left[\left|d_{2}\right| \delta_{\Omega}^{-1}\left\|\left(1-\xi^{2}\right)^{\frac{r-2}{2}}\left(a_{1}+a_{3} \eta\right)^{j}\left(b_{1}+b_{3} \eta\right)^{k-j} \partial_{x}^{j} \partial_{y}^{k-j} v\right\|_{\Omega}\right. \\
+\gamma_{\Omega}\left\|\left(1-\xi^{2}\right)^{\frac{r-2}{2}}\left(a_{1}+a_{3} \eta\right)_{\dagger}^{j-1}\left(b_{1}+b_{3} \eta\right)^{k-j} \partial_{x}^{j} \partial_{y}^{k-j} v\right\|_{\Omega} \\
+\gamma_{\Omega}\left\|\left(1-\xi^{2}\right)^{\frac{r-2}{2}}\left(a_{1}+a_{3} \eta\right)^{j}\left(b_{1}+b_{3} \eta\right)_{\dagger}^{k-j-1} \partial_{x}^{j} \partial_{y}^{k-j} v\right\|_{\Omega} \\
+\sigma_{\Omega}\left\|\left(1-\xi^{2}\right)^{\frac{r-2}{2}}\left(a_{1}+a_{3} \eta\right)^{j}\left(b_{1}+b_{3} \eta\right)^{k-j} \partial_{x}^{j+1} \partial_{y}^{k-j} v\right\|_{\Omega} \\
\left.+\sigma_{\Omega}\left\|\left(1-\xi^{2}\right)^{\frac{r-2}{2}}\left(a_{1}+a_{3} \eta\right)^{j}\left(b_{1}+b_{3} \eta\right)^{k-j} \partial_{x}^{j} \partial_{y}^{k-j+1} v\right\|_{\Omega}\right]
\end{aligned}
$$


and

$$
\begin{aligned}
\| \partial_{\xi} \partial_{\eta}^{r-1} u & \left.\|_{L^{2}\left(\Lambda_{\xi}, L_{\chi}^{2}(r-2, r-2)\right.}\left(\Lambda_{\eta}\right)\right) \leq c \sum_{k=0}^{r-1} \sum_{j=0}^{k}\left|d_{2}\right|^{r-k-1} \delta_{\Omega}^{-r+k+1} \\
& \cdot\left[\left|d_{1}\right| \delta_{\Omega}^{-1}\left\|\left(1-\eta^{2}\right)^{\frac{r-2}{2}}\left(a_{2}+a_{3} \xi\right)^{j}\left(b_{2}+b_{3} \xi\right)^{k-j} \partial_{x}^{j} \partial_{y}^{k-j} v\right\|_{\Omega}\right. \\
& +\gamma_{\Omega}\left\|\left(1-\eta^{2}\right)^{\frac{r-2}{2}}\left(a_{2}+a_{3} \xi\right)_{\dagger}^{j-1}\left(b_{2}+b_{3} \xi\right)^{k-j} \partial_{x}^{j} \partial_{y}^{k-j} v\right\|_{\Omega} \\
& +\gamma_{\Omega}\left\|\left(1-\eta^{2}\right)^{\frac{r-2}{2}}\left(a_{2}+a_{3} \xi\right)^{j}\left(b_{2}+b_{3} \xi\right)_{\dagger}^{k-j-1} \partial_{x}^{j} \partial_{y}^{k-j} v\right\|_{\Omega} \\
& +\sigma_{\Omega}\left\|\left(1-\eta^{2}\right)^{\frac{r-2}{2}}\left(a_{2}+a_{3} \xi\right)^{j}\left(b_{2}+b_{3} \xi\right)^{k-j} \partial_{x}^{j+1} \partial_{y}^{k-j} v\right\|_{\Omega} \\
& \left.+\sigma_{\Omega}\left\|\left(1-\eta^{2}\right)^{\frac{r-2}{2}}\left(a_{2}+a_{3} \xi\right)^{j}\left(b_{2}+b_{3} \xi\right)^{k-j} \partial_{x}^{j} \partial_{y}^{k-j+1} v\right\|_{\Omega}\right] .
\end{aligned}
$$

Then, the first result of (3.30) comes from a combination of (3.31)-(3.35), (3.38) and (3.39).

We now prove the second result of (3.30). Let $g \in L^{2}(\Omega)$ and consider an auxiliary problem which is to find $w \in H_{0}^{1}(\Omega)$ such that

$$
(\nabla w, \nabla z)_{\Omega}=(g, z)_{\Omega}, \forall z \in H_{0}^{1}(\Omega)
$$

Taking $z=w$ in (3.40) and using (3.28), we obtain $\|\nabla w\|_{\Omega} \leq c_{\Omega}\|g\|_{\Omega}$. Moreover, by the property of the elliptic equation with the homogeneous boundary condition, there exists a positive constant $\bar{c}_{\Omega}$ such that

$$
\begin{aligned}
\|w\|_{H^{2}(\Omega)} & \leq \bar{c}_{\Omega}\left(\|w\|_{\Omega}+\|g\|_{\Omega}\right) \leq \bar{c}_{\Omega}\left(c_{\Omega}\|\nabla w\|_{\Omega}+\|g\|_{\Omega}\right) \\
& \leq \bar{c}_{\Omega}\left(c_{\Omega}^{2}+1\right)\|g\|_{\Omega} .
\end{aligned}
$$

Taking $z=P_{N}^{1,0} v-v$ in (3.40). Then we use (3.29) and the first result of (3.30) to obtain

$$
\begin{aligned}
\left|\left(P_{N}^{1,0} v-v, g\right)_{\Omega}\right| & =\left|\left(\nabla w, \nabla\left(P_{N}^{1,0} v-v\right)\right)_{\Omega}\right|=\left|\left(\nabla\left(P_{N}^{1,0} w-w\right), \nabla\left(P_{N}^{1,0} v-v\right)\right)_{\Omega}\right| \\
& \leq\left\|\nabla\left(P_{N}^{1,0} w-w\right)\right\|_{\Omega}\left\|\nabla\left(P_{N}^{1,0} v-v\right)\right\|_{\Omega} \leq c N^{-r} B_{r, \Omega}(v) B_{2, \Omega}(w) .
\end{aligned}
$$

Since $r \geq 2$, we use (3.26) to assert that $B_{2, \Omega}(w) \leq \beta_{\Omega}\|w\|_{H^{2}(\Omega)}$ where

$$
\begin{gathered}
\beta_{\Omega}=\left(\left(\left|d_{1}\right|+\left|d_{2}\right|\right) \delta_{\Omega}^{-1}+1\right)\left(\sum_{k=0}^{r} \delta_{\Omega}^{k-3} \sigma_{\Omega}^{k}(k+1)\left(\left|d_{1}\right|^{2-k}+\left|d_{2}\right|^{2-k} \mid\right)\right. \\
+\sum_{k=0}^{r-1} \delta_{\Omega}^{k-2} \sigma_{\Omega}^{k}(k+1)\left[\left|d_{1}\right|^{1-k}\left(\left|d_{2}\right| \delta_{\Omega}^{-1}+\gamma_{\Omega} \sigma_{\Omega}+\sigma_{\Omega}\right)\right. \\
\left.\left.+\left|d_{2}\right|^{1-k}\left(\left|d_{1}\right| \delta_{\Omega}^{-1}+\gamma_{\Omega} \sigma_{\Omega}+\sigma_{\Omega}\right)\right]\right) .
\end{gathered}
$$

Finally, we have from (3.41) and (3.42) that

$$
\begin{aligned}
\left\|P_{N}^{1,0} v-v\right\|_{\Omega} & =\sup _{g \in L^{2}(\Omega), g \neq 0} \frac{\left|\left(P_{N}^{1,0} v-v, g\right)_{\Omega}\right|}{\|g\|_{\Omega}} \leq c N^{-r} \frac{B_{r, \Omega}(v) B_{2, \Omega}(w)}{\|g\|_{\Omega}} \\
& \leq c \beta_{\Omega} \frac{B_{r, \Omega}(v)\|w\|_{H^{2}(\Omega)}}{\|g\|_{\Omega}} \leq c \bar{c}_{\Omega} \beta_{\Omega} N^{-r} B_{r, \Omega}(v) .
\end{aligned}
$$

This ends the proof.

Remark 3.4. By the definition of $B_{r, \Omega}(v)$ and (3.24), there are the weight functions $\left|\left(1-\xi^{2}\right)^{r-k-2}\left(\left(x-x_{1}^{*}\right)\left(x-x_{3}^{*}\right)\right)^{j}\left(\left(y-y_{1}^{*}\right)\left(y-y_{3}^{*}\right)\right)^{k-j}\right|$ and $\mid\left(1-\eta^{2}\right)^{r-k-2}((x-$ $\left.\left.x_{2}^{*}\right)\left(x-x_{4}^{*}\right)\right)^{j}\left(\left(y-y_{2}^{*}\right)\left(y-y_{4}^{*}\right)\right)^{k-j} \mid$, in the norm of derivative $\partial_{x}^{j} \partial_{y}^{k-j} v$, etc. Since $r \geq \max (k, 2)$, they decay to zero as the point $Q(x, y)$ goes to the corners of the quadrilateral. Therefore, the error of $H_{0}^{1}(\Omega)$-orthogonal approximation keeps the order $N^{1-r}$, even if the considered function possesses certain singularities at 
the vertices of the quadrilateral. For instance, $\left|\partial_{x}^{j} \partial_{y}^{k-j} v\right|=\mathrm{o}\left(\left(1-\xi^{2}\right)^{\frac{-r+k+2}{2}} \mid Q-\right.$ $\left.\left.Q_{\nu}^{*}\right|^{\frac{-2 k-1}{2}}\right)$ as $Q(x, y)$ tends to the edges $L_{1}$ or $L_{3}$, etc.

Remark 3.5. If $\Omega=\{(x, y)|| x|<a| y \mid,<b, a, b,>0\}$, then $\sigma_{\Omega}=\max (a, b), \delta_{\Omega}=a b$ and $d_{1}=d_{2}=\gamma_{\Omega}=0$. Moreover, $a_{1}=a, b_{2}=b$ and $a_{2}=a_{3}=b_{1}=b_{3}=0$. Thus, we have from (3.36) and (3.37) that

$$
\partial_{\xi}^{r-1} \partial_{\eta} u=a^{r-\frac{1}{2}} b^{\frac{3}{2}} \partial_{x}^{r-1} \partial_{y} v, \quad \partial_{\xi} \partial_{\eta}^{r-1} u=a^{\frac{3}{2}} b^{r-\frac{1}{2}} a^{\frac{3}{2}} \partial_{x} \partial_{y}^{r-1} v .
$$

Accordingly, the quantity $B_{r, \Omega}(v)$ is reduced to

$$
\begin{aligned}
B_{r, \Omega}(v) & =\max (a, b)\left(b^{-1}\left\|\left(a^{2}-x^{2}\right)^{\frac{r-1}{2}} \partial_{x}^{r} v\right\|_{\Omega}+a^{-1}\left\|\left(b^{2}-y^{2}\right)^{\frac{r-1}{2}} \partial_{y}^{r} v\right\|_{\Omega}\right) \\
& +a b \max (a, b)\left(\left\|\left(a^{2}-x^{2}\right)^{\frac{r-2}{2}} \partial_{x}^{r-1} \partial_{y} v\right\|_{\Omega}+\left\|\left(b^{2}-y^{2}\right)^{\frac{r-2}{2}} \partial_{x} \partial_{y}^{r-1} v\right\|_{\Omega}\right) .
\end{aligned}
$$

Therefore, the error of the $H_{0}^{1}(\Omega)$-orthogonal approximation still keeps the order $N^{1-r}$, even if the considered function possesses certain singularities at the edges of the quadrilateral. If, in addition, $a=b=1$, then the above estimate turns out to be the same result as in [13.

\section{Spectral Method for QUAdrilaterals}

In this section, we propose the spectral method for quadrilaterals.

4.1. A steady problem. As an example, we consider the following simple problem:

$$
\begin{cases}-\Delta U(x, y)=f(x, y), & (x, y) \in \Omega \\ U(x, y)=g(x, y), & (x, y) \in \partial \Omega .\end{cases}
$$

We first consider the case $g(x, y) \equiv 0$. Then, the weak formulation of (4.1) is to seek solution $U \in H_{0}^{1}(\Omega)$, such that

$$
(\nabla U, \nabla v)_{\Omega}=(f, v)_{\Omega}, \quad \forall v \in H_{0}^{1}(\Omega) .
$$

The spectral scheme for (4.2) is to find $u_{N} \in V_{N}^{0}(\Omega)$, such that

$$
\left(\nabla u_{N}, \nabla \phi\right)_{\Omega}=(f, \phi)_{\Omega}, \quad \forall \phi \in V_{N}^{0}(\Omega) .
$$

For the convergence analysis, we set $U_{N}=P_{N}^{1,0} U$. We have from (4.2) with (3.29) that

$$
\left(\nabla U_{N}, \nabla \phi\right)_{\Omega}=(f, \phi)_{\Omega}, \quad \forall \phi \in V_{N}^{0}(\Omega) .
$$

Let $\widetilde{U}_{N}=u_{N}-U_{N}$. Subtracting (4.4) from (4.3), we obtain

$$
\left(\nabla \widetilde{U}_{N}, \nabla \phi\right)_{\Omega}=0, \quad \forall \phi \in V_{N}^{0}(\Omega) .
$$

Taking $\phi=\widetilde{U}_{N}$ in the above, we get $\left\|\nabla \widetilde{U}_{N}\right\|_{\Omega}=0$. This fact with the Poincaré inequality on $\Omega$ implies $\widetilde{U}_{N}=0$, i.e., $u_{N}=P_{N}^{1,0} U$. Finally, by (3.30),

$$
\left\|\nabla\left(u_{N}-U\right)\right\|_{\Omega} \leq c N^{1-r} B_{r, \Omega}(U), \quad\left\|u_{N}-U\right\|_{\Omega} \leq c c_{\Omega}^{*} N^{-r} B_{r, \Omega}(U) .
$$

Remark 4.1. In practice, $U(x, y)$ might not vanish on the boundary. In this case, we construct a function $W(x, y)$ which equals $U(x, y)$ on $\partial \Omega$; see (7.3) of this paper. Then, we solve the corresponding problem and obtain the numerical solution of original problem. 
Remark 4.2. The spectral scheme (4.4) is similar to scheme (1.15) of [2]. But scheme (1.15) of [2] is a pseudospectral method. Our method is more similar to scheme (5.3.11) of [5] for a single domain, as well as the related method in 17. Whereas they are not exactly the same. First, one uses the base functions $L_{l}(\xi(x, y)) L_{m}(\eta(x, y))$ in [2, 5, 17, which are not mutually orthogonal in the space $L^{2}(\Omega)$. However, we use the base functions $L_{l}(\xi(x, y)) L_{m}(\eta(x, y)) J_{S}^{\frac{1}{2}}(x, y)$, which are mutually orthogonal in the space $L^{2}(\Omega)$. It is more natural. As a result, we could use the recent result on the Jacobi orthogonal approximation to derive better error estimate (3.30) of numerical solution, with the Jacobi weights, which cover certain singularities on the vertices of $\Omega$.

4.2. An unsteady problem. Let $\beta$ be a constant. We consider the problem (4.7)

$\begin{cases}\partial_{t} U(x, y, t)=\Delta U(x, y, t)+\beta U(x, y, t)+f(x, y, t), & (x, y) \in \Omega, 0<t \leq T, \\ U(x, y, t)=g(x, y, t), & (x, y) \in \partial \Omega, 0<t \leq T, \\ U(x, y, 0)=U_{0}(x, y), & (x, y) \in \Omega \cup \partial \Omega .\end{cases}$

We first consider the case with $g(x, y, t)=0$. Then, the weak formulation of (4.7) is to find $U \in L^{\infty}\left(0, T ; L^{2}(\Omega)\right) \cap L^{2}\left(0, T ; H_{0}^{1}(\Omega)\right)$, such that

$$
\begin{cases}\left(\partial_{t} U(t), v\right)_{\Omega}+(\nabla U(t), \nabla v)_{\Omega}= & \beta(U(t), v)_{\Omega}+(f(t), v)_{\Omega}, \\ U(0)=U_{0} . & \forall v \in H_{0}^{1}(\Omega), 0<t \leq T,\end{cases}
$$

The spectral scheme for (4.8) is to find $u_{N} \in V_{N}^{0}(\Omega)$ for all $0<t \leq T$, such that

$$
\left\{\begin{array}{rr}
\left(\partial_{t} u_{N}(t), \phi\right)_{\Omega}+\left(\nabla u_{N}(t), \nabla \phi\right)_{\Omega}=\beta\left(u_{N}(t), v\right)_{\Omega}+(f(t), \phi)_{\Omega}, & \\
u_{N}(0)=P_{N} U_{0} . & \forall \phi \in V_{N}^{0}(\Omega), 0<t \leq T,
\end{array}\right.
$$

We now analyze the convergence. Letting $U_{N}=P_{N}^{1,0} U$, we obtain from (4.8) that

$$
\left\{\begin{array}{l}
\left(\partial_{t} U_{N}(t), \phi\right)_{\Omega}+\left(\nabla U_{N}(t), \nabla \phi\right)_{\Omega}=\beta\left(U_{N}(t), v\right)_{\Omega} \\
\quad+(f(t), \phi)_{\Omega}+G_{1}(t, \phi)+G_{2}(t, \phi), \forall \phi \in V_{N}^{0}(\Omega), 0<t \leq T \\
U_{N}(0)=P_{N}^{1,0} U_{0}
\end{array}\right.
$$

with

$$
G_{1}(t, \phi)=\left(\partial_{t}\left(U_{N}(t)-U(t)\right), \phi\right)_{\Omega}, \quad G_{2}(t, \phi)=\beta\left(U(t)-U_{N}(t), \phi\right)_{\Omega} .
$$

Letting $\widetilde{U}_{N}=u_{N}-U_{N}$, and subtracting (4.10) from (4.9), we obtain

$$
\left\{\begin{array}{lr}
\left(\partial_{t} \widetilde{U}_{N}, \phi\right)_{\Omega}+\left(\nabla \widetilde{U}_{N}, \nabla \phi\right)_{\Omega}=\beta\left(\widetilde{U}_{N}(t), v\right)_{\Omega}-G_{1}(t, \phi)-G_{2}(t, \phi), \\
\widetilde{U}_{N}(0)=P_{N} U_{0}-P_{N}^{1,0} U_{0} & \forall \phi \in V_{N}^{0}(\Omega), 0<t \leq T,
\end{array}\right.
$$

Take $\phi=2 \widetilde{U}_{N}$ in (4.11). Then,

$$
\partial_{t}\left\|\widetilde{U}_{N}(t)\right\|_{\Omega}^{2}+2\left\|\nabla \widetilde{U}_{N}(t)\right\|_{\Omega}^{2} \leq 2 \beta\left\|\widetilde{U}_{N}(t)\right\|_{\Omega}^{2}+2\left|G_{1}\left(t, \widetilde{U}_{N}\right)\right|+2\left|G_{2}\left(t, \widetilde{U}_{N}\right)\right| .
$$

By virtue of (3.28) and (3.30), we have

$$
\begin{aligned}
2\left|G_{1}\left(t, \widetilde{U}_{N}\right)\right| & \leq \frac{1}{2 c_{\Omega}^{2}}\left\|\widetilde{U}_{N}(t)\right\|_{\Omega}^{2}+2 c_{\Omega}^{2}\left\|\partial_{t}\left(U_{N}(t)-U(t)\right)\right\|_{\Omega}^{2} \\
& \leq \frac{1}{2}\left\|\nabla \widetilde{U}_{N}(t)\right\|_{\Omega}^{2}+c c_{\Omega}^{2}\left(c_{\Omega}^{*}\right)^{2} N^{-2 r} B_{r, \Omega}^{2}\left(\partial_{t} U(t)\right) .
\end{aligned}
$$


Similarly,

$$
2\left|G_{2}\left(t, \widetilde{U}_{N}\right)\right| \leq \frac{1}{2}\left\|\nabla \widetilde{U}_{N}\right\|_{\Omega}^{2}+c \beta^{2} c_{\Omega}^{2}\left(c_{\Omega}^{*}\right)^{2} N^{-2 r} B_{r, \Omega}^{2}(U(t)) .
$$

For simplicity of statements, let

$$
E(v(t))=\|v(t)\|_{\Omega}^{2}+\int_{0}^{t}\|\nabla v(\xi)\|_{\Omega}^{2} d \xi .
$$

Substituting (4.13) and (4.14) into (4.12), we obtain

$$
\partial_{t}\left(E\left(\widetilde{U}_{N}(t)\right) e^{-2 \beta t}\right) \leq c e^{-2 \beta t}\left(\beta^{2}+1\right) c_{\Omega}^{2}\left(c_{\Omega}^{*}\right)^{2} N^{-2 r}\left(B_{r, \Omega}^{2}\left(\partial_{t} U(t)\right)+B_{r, \Omega}^{2}(U(t))\right) .
$$

On the other hand, we use (3.10) and the second result of (3.30) to obtain

$$
\begin{aligned}
\left\|\widetilde{U}_{N}(0)\right\|_{\Omega}^{2} & \leq 2\left\|P_{N} U_{0}-U_{0}\right\|_{\Omega}^{2}+2\left\|U_{0}-P_{N}^{1,0} U_{0}\right\|_{\Omega}^{2} \\
& \leq c N^{-2 r}\left(A_{r, \Omega}^{2}\left(U_{0}\right)+\left(c_{\Omega}^{*}\right)^{2} B_{r, \Omega}^{2}\left(U_{0}\right)\right) .
\end{aligned}
$$

Integrating (4.15) with respect to $t$ and using (4.16), we obtain

$$
E\left(\widetilde{U}_{N}(t)\right) \leq c N^{-2 r} e^{2 \beta t} R_{r, \Omega, \beta}(U, t)
$$

where

$$
\begin{aligned}
R_{r, \Omega, \beta}(U, t)=\left(\beta^{2}+1\right) c_{\Omega}^{2}\left(c_{\Omega}^{*}\right)^{2} \int_{0}^{t} e^{-2 \beta \xi}\left(B_{r, \Omega}^{2}\left(\partial_{\xi} U(\xi)\right)+B_{r, \Omega}^{2}(U(\xi))\right) d \xi \\
+A_{r, \Omega}^{2}\left(U_{0}\right)+\left(c_{\Omega}^{*}\right)^{2} B_{r, \Omega}^{2}\left(U_{0}\right) .
\end{aligned}
$$

Finally, a combination of (3.30) and (4.17) leads to

$$
\begin{gathered}
\int_{0}^{t}\left\|\nabla\left(u_{N}(\xi)-U(\xi)\right)\right\|_{\Omega}^{2} d \xi \leq c N^{-2 r} e^{2 \beta t} R_{r, \Omega, \beta}(U, t) \\
+c N^{2-2 r} \int_{0}^{t} B_{r, \Omega}^{2}(U(\xi)) d \xi, \\
\left\|u_{N}(t)-U(t)\right\|_{\Omega}^{2} \leq c N^{-2 r}\left(e^{2 \beta t} R_{r, \Omega, \beta}(U, t)+\left(c_{\Omega}^{*}\right)^{2} B_{r, \Omega}^{2}(U(t))\right) .
\end{gathered}
$$

\section{NUMERICAL imPLEMENTATION}

In this section, we describe numerical implementation. Let

$$
\sigma_{l}(\zeta)=c_{l}\left(L_{l}(\zeta)-L_{l+2}(\zeta)\right), \quad c_{l}=(4 l+6)^{-\frac{1}{2}} .
$$

Clearly, $\sigma_{l}( \pm 1)=0$. Moreover, a calculation shows

$$
\partial_{\zeta} \sigma_{l}(\zeta)=d_{l} L_{l}(\zeta), \quad d_{l}=2^{-\frac{1}{2}}(l+1)^{-1}(2 l+3)^{\frac{3}{2}} .
$$

Further, we set

$$
\psi_{l, m}(x, y)=\sigma_{l}(\xi(x, y)) \sigma_{m}(\eta(x, y)) J_{S}^{\frac{1}{2}}(x, y), \quad 0 \leq l, m \leq N-2,
$$

which form a basis of $V_{N}^{0}(\Omega)$. 
We first consider the scheme (4.3). We expand the numerical solution $u_{N}(x, y)$ as

$$
u_{N}(x, y)=\sum_{k, j=0}^{N-2} u_{N, k, j} \psi_{k, j}(x, y) .
$$

Substituting (5.4) into (4.3) and putting $\phi(x, y)=\psi_{l, m}(x, y)$ in the resulting equation, we derive a linear system with the unknown coefficients $u_{N, k, j}, 0 \leq k, j \leq$ $N-2$. This system can be rewritten as a compact matrix form. To do this, let

$$
\begin{aligned}
& \mathbf{u}=\left(u_{N, 0,0}, u_{N, 0,1}, \cdots u_{N, 0, N-2}, \cdots u_{N, N-2,0}, \cdots u_{N, N-2, N-2}\right)^{T}, \\
& \mathbf{F}=\left(f_{0,0}, f_{0,1}, \cdots f_{0, N-2}, \cdots f_{N-2,0}, \cdots f_{N-2, N-2}\right)^{T}, \quad f_{l, m}=\left(f, \psi_{l, m}\right)_{\Omega} .
\end{aligned}
$$

Furthermore, let $a_{l, m, k, j}=\left(\partial_{x} \psi_{k, j}, \partial_{x} \psi_{l, m}\right)_{\Omega}+\left(\partial_{y} \psi_{k, j}, \partial_{y} \psi_{l, m}\right)_{\Omega}$. The matrix

$$
A=\left(\begin{array}{cccccc}
a_{0,0,0,0} & a_{0,0,0,1} & \cdots & a_{0,0, N-2,0} & \cdots & a_{0,0, N-2, N-2} \\
a_{0,1,0,0} & a_{0,1,0,1} & \cdots & a_{0,1, N-2,0} & \cdots & a_{0,1, N-2, N-2} \\
\cdots & \cdots & \cdots & \cdots & \cdots & \cdots \\
a_{N-2, N-2,0,0} & a_{N-2, N-2,0,1} & \cdots & a_{N-2, N-2, N-2,0} & \cdots & a_{N-2, N-2, N-2, N-2}
\end{array}\right) .
$$

Consequently, we have

$$
A \mathbf{u}=\mathbf{F} .
$$

The matrix $A$ is full. But its condition number (labeled by Cond.) is acceptable; see Table 5.1.

TABLE 5.1. Condition number of matrix $A$.

\begin{tabular}{|c|c|c|c|c|c|}
\hline $\mathrm{N}$ & 5 & 10 & 15 & 20 & 25 \\
\hline Cond. & 34.3 & 410.5 & 1873.6 & 5624.1 & 13316.2 \\
\hline
\end{tabular}

We now turn to (4.8). We approximate the term $\partial_{t} u_{N}(t)$ by the Crank-Nicholson discretization with the mesh size $\tau$. It is of the form (5.6)

$$
\begin{aligned}
\frac{1}{\tau}\left(u_{N}(t\right. & \left.+\tau)-u_{N}(t), \psi_{l, m}\right)_{\Omega}+\frac{1}{2}\left(\partial_{x}\left(u_{N}(t+\tau)\right)+\partial_{x} u_{N}(t), \partial_{x} \psi_{l, m}\right)_{\Omega} \\
& +\frac{1}{2}\left(\partial_{y}\left(u_{N}(t+\tau)\right)+\partial_{y} u_{N}(t), \partial_{y} \psi_{l, m}\right)_{\Omega} \\
& =\frac{1}{2} \beta\left(u_{N}(t+\tau)+u_{N}(t), \psi_{l, m}\right)_{\Omega}+f_{l, m}\left(t+\frac{\tau}{2}\right), \quad 0 \leq l, m \leq N-2,
\end{aligned}
$$

where $f_{l, m}\left(t+\frac{\tau}{2}\right)=\left(f\left(t+\frac{\tau}{2}\right), \psi_{l, m}\right)_{\Omega}$. We expand the numerical solution as

$$
u_{N}(x, y, t)=\sum_{k, j=0}^{N-2} u_{N, k, j}(t) \psi_{k, j}(x, y) .
$$

Inserting (5.7) into (5.6), we obtain a scheme for the unknown coefficients $u_{N, k, j}(t)$, $t=\tau, 2 \tau, \cdots,\left[\frac{T}{\tau}\right]$. It can be also written as a compact matrix form. To do this, let

$$
\begin{gathered}
\mathbf{u}(t)=\left(u_{N, 0,0}(t), u_{N, 0,1}(t), \cdots, u_{N, N-2, N-2}(t)\right)^{T} \\
\mathbf{F}\left(t+\frac{\tau}{2}\right)=\left(f_{0,0}\left(t+\frac{\tau}{2}\right), f_{0,1}\left(t+\frac{\tau}{2}\right), \cdots, f_{N-2, N-2}\left(t+\frac{\tau}{2}\right)\right)^{T} .
\end{gathered}
$$

Let $A$ be the same matrix as in (5.5). The matrix $B$ is similar to $A$, with the entries $b_{l, m, k, j}=\left(\psi_{k, j}, \psi_{l, m}\right)_{\Omega}$. Then, the matrix form of (5.6) is

$$
(\tau A+(2-\tau \beta) B) \mathbf{u}(t+\tau)=(-\tau A+(2+\tau \beta) B) \mathbf{u}(t)+2 \tau \mathbf{F}\left(t+\frac{\tau}{2}\right) .
$$




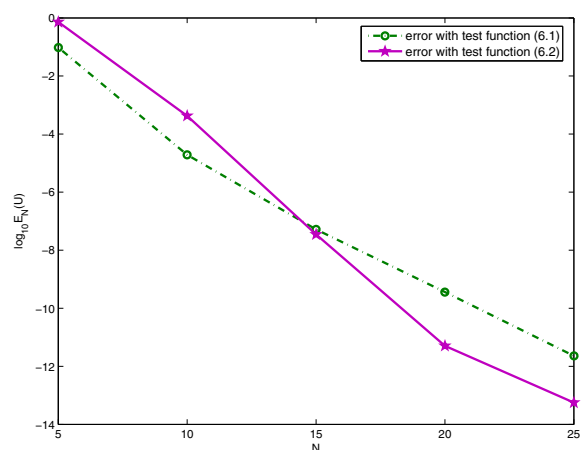

FIgURE 3. Numerical errors of (4.3).

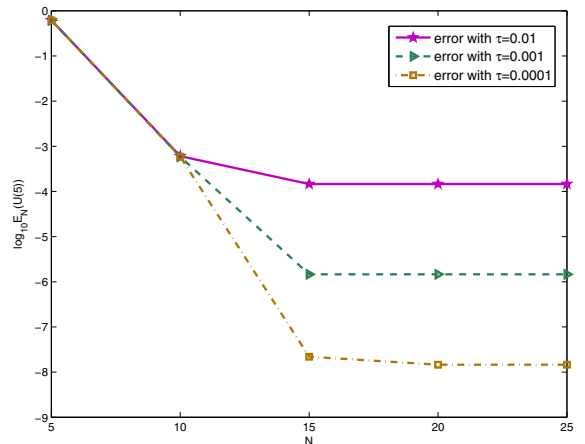

FiguRE 5. Numerical errors of (5.6).

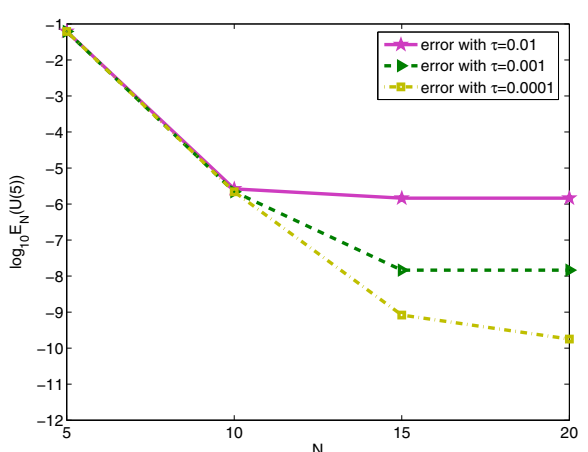

FiguRE 4. Numerical errors of (5.6).

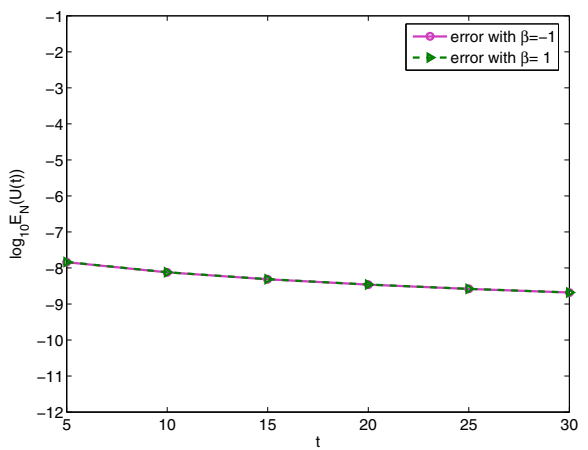

FiguRE 6. Stability of (5.6).

\section{NumericAl RESUlts}

We first use (4.3) to solve (4.2) with the test function (6.1)

$$
\begin{aligned}
U(x, y)= & \left(y-\frac{\left(x-x_{1}\right)\left(y_{4}-y_{1}\right)}{\left(x_{4}-x_{1}\right)}-y_{1}\right)\left(y-\frac{\left(x-x_{2}\right)\left(y_{2}-y_{1}\right)}{\left(x_{2}-x_{1}\right)}-y_{2}\right)\left(y-\frac{\left(x-x_{3}\right)\left(y_{3}-y_{2}\right)}{\left(x_{3}-x_{2}\right)}-y_{3}\right) \\
& \left(y-\frac{\left(x-x_{4}\right)\left(y_{4}-y_{3}\right)}{\left(x_{4}-x_{3}\right)}-y_{4}\right) \sqrt{x^{2}+y^{2}+1}
\end{aligned}
$$

where $x_{j}$ and $y_{j}, 1 \leq j \leq 4$ are the coordinates of the vertices of $\Omega$. We shall also use the test function

$$
U(x, y)=4 \sin (2 x+3 y)
$$

which does not vanish on the boundary. In actual calculation, we take $x_{1}=0, x_{2}=$ $3, x_{3}=2, x_{4}=1, y_{1}=0, y_{2}=0, y_{3}=3$ and $y_{4}=2$. For description of numerical 
errors, let $\zeta_{M, i}$ and $\omega_{M, i}$ be the nodes and the weights of one-dimensional LegendreGauss-Lobatto quadrature. We measure the numerical errors by

$$
\begin{aligned}
& E_{N}(U)=\left(\sum _ { i , j = 1 } ^ { M } \left(U\left(x\left(\zeta_{M, i}, \zeta_{M, j}\right), y\left(\zeta_{M, i}, \zeta_{M, j}\right)\right)\right.\right. \\
& \left.\left.\quad-u_{N}\left(x\left(\zeta_{M, i}, \zeta_{M, j}\right), y\left(\zeta_{M, i}, \zeta_{M, j}\right)\right)\right)^{2}\left(d_{0}+d_{1} \zeta_{M, i}+d_{2} \zeta_{M, j}\right) \omega_{M, i} \omega_{M, j}\right)^{\frac{1}{2}} \\
& \approx\left(\iint_{S}\left(U(x(\xi, \eta), y(\xi, \eta))-u_{N}(x(\xi, \eta), y(\xi, \eta))\right)^{2} J_{\Omega}(\xi, \eta) d \xi d \eta\right)^{\frac{1}{2}}=\left\|U-u_{N}\right\|_{\Omega} .
\end{aligned}
$$

In Figure 3, we plot the values of $\log _{10} E_{N}(U)$ vs. the mode $N$, with the test functions (6.1) and (6.2). They demonstrate the high accuracy of scheme (4.3). (6.3)

We next use (4.9) to solve (4.8), with the test function

$$
\begin{aligned}
U(x, y, t)= & \left(y-\frac{\left(x-x_{1}\right)\left(y_{4}-y_{1}\right)}{\left(x_{4}-x_{1}\right)}-y_{1}\right)\left(y-\frac{\left(x-x_{2}\right)\left(y_{2}-y_{1}\right)}{\left(x_{2}-x_{1}\right)}-y_{2}\right)\left(y-\frac{\left(x-x_{3}\right)\left(y_{3}-y_{2}\right)}{\left(x_{3}-x_{2}\right)}-y_{3}\right) \\
& \left(y-\frac{\left(x-x_{4}\right)\left(y_{4}-y_{3}\right)}{\left(x_{4}-x_{3}\right)}-y_{4}\right) \sqrt{x^{2}+y^{2}+t+1} .
\end{aligned}
$$

For describing the numerical errors at a different time $t$, we use the notation $E_{N}(U(t))$. In Figure 4, we plot the values of $E_{N}(U(t))$ with $\beta=1, t=5, N=$ $5,10,15,20$, and $\tau=0.01,0.001,0.0001$. Clearly, the error decays rapidly as $N$ increases and $\tau$ decreases. It also shows the high accuracy in space. We also use (4.12) to solve (4.11), with the test function

$$
U(x, y)=4 \sin (2 x+3 y+t) .
$$

In Figure 5, we plot the corresponding values of $E_{N}(U(t))$ with $\beta=1$. They indicate again the high accuracy in space.

In Figure 6, we plot the values of $E_{N}(U(t))$ with the test function (6.3), $\beta=$ $\pm 1, N=20$ and $\tau=0.001$. They demonstrate the stability of (5.6).

\section{THE SPECTRAL METHOD FOR POLYGONS}

In this section, we investigate the spectral method for polygons.

7.1. Some preparations. We first consider several quasi-Legendre orthogonal approximations on the convex quadrilateral $\Omega$. To do this, we put $\hat{v}(\xi, \eta)=$ $v(x(\xi, \eta), y(\xi, \eta))$, and

$$
\begin{aligned}
& \hat{v}_{b, L_{1}}(\eta)=\frac{1}{2}(\hat{v}(-1,-1)(1-\eta)+\hat{v}(-1,1)(1+\eta)), \\
& \hat{v}_{b, L_{2}}(\xi)=\frac{1}{2}(\hat{v}(-1,-1)(1-\xi)+\hat{v}(1,-1)(1+\xi)), \\
& \hat{v}_{b, L_{3}}(\eta)=\frac{1}{2}(\hat{v}(1,-1)(1-\eta)+\hat{v}(1,1)(1+\eta)), \\
& \hat{v}_{b, L_{4}}(\xi)=\frac{1}{2}(\hat{v}(-1,1)(1-\xi)+\hat{v}(1,1)(1+\xi)) .
\end{aligned}
$$

Next, we set

$$
\begin{array}{ll}
\hat{v}_{b, L_{1}}^{0}(\eta)=\hat{v}(-1, \eta)-\hat{v}_{b, L_{1}}(\eta), & \hat{v}_{b, L_{2}}^{0}(\xi)=\hat{v}(\xi,-1)-\hat{v}_{b, L_{2}}(\xi), \\
\hat{v}_{b, L_{3}}^{0}(\eta)=\hat{v}(1, \eta)-\hat{v}_{b, L_{3}}(\eta), & \hat{v}_{b, L_{4}}^{0}(\xi)=\hat{v}(\xi, 1)-\hat{v}_{b, L_{4}}(\xi) .
\end{array}
$$


The above four functions vanish at the endpoints of $\Lambda_{\xi}$ or $\Lambda_{\eta}$, respectively. We also set

$$
\begin{aligned}
\hat{v}_{b, \partial \Omega}(\xi, \eta)= & \hat{v}_{b, \partial \Omega}^{(1)}(\xi, \eta)+\hat{v}_{b, \partial \Omega}^{(2)}(\xi, \eta), \\
\hat{v}_{b, \partial \Omega}^{(1)}(\xi, \eta)= & \frac{1}{2}((1-\xi) \hat{v}(-1, \eta)+(1-\eta) \hat{v}(\xi,-1)+(1+\xi) \hat{v}(1, \eta)+(1+\eta) \hat{v}(\xi, 1)), \\
\hat{v}_{b, \partial \Omega}^{(2)}(\xi, \eta)= & -\frac{1}{4}((1-\xi)(1-\eta) \hat{v}(-1,-1)+(1+\xi)(1-\eta) \hat{v}(1,-1) \\
& +(1+\xi)(1+\eta) \hat{v}(1,1)+(1-\xi)(1+\eta) \hat{v}(-1,1)),
\end{aligned}
$$

or equivalently,

$$
\begin{aligned}
\hat{v}_{b, \partial \Omega}(\xi, \eta)= & \frac{1}{2}\left((1-\xi) \hat{v}_{b, L_{1}}^{0}(\eta)+(1-\eta) \hat{v}_{b, L_{2}}^{0}(\xi)+(1+\xi) \hat{v}_{b, L_{3}}^{0}(\eta)+(1+\eta) \hat{v}_{b, L_{4}}^{0}(\xi)\right) \\
& +\frac{1}{4}((1-\xi)(1-\eta) \hat{v}(-1,-1)+(1+\xi)(1-\eta) \hat{v}(1,-1) \\
& +(1+\xi)(1+\eta) \hat{v}(1,1)+(1-\xi)(1+\eta) \hat{v}(-1,1)) .
\end{aligned}
$$

Let $\hat{v}_{\Omega}^{0}(\xi, \eta)=\hat{v}(\xi, \eta)-\hat{v}_{b, \partial \Omega}(\xi, \eta)$. Obviously, $\hat{v}_{\Omega}^{0}(\xi, \eta)$ vanishes on $\partial \Omega$.

For the spectral method for a union of quadrilaterals, it is simpler to take the base functions $\psi_{l, m}(x, y)=L_{l}(\xi(x, y)) L_{m}(\eta(x, y))$, as in 2, 5, 17. Let $V_{N}(\Omega)=$ $\left\{\psi_{l, m}(x, y), 0 \leq l, m \leq N\right\}$ and $V_{N}^{0}(\Omega)=H_{0}^{1}(\Omega) \cap V_{N}(\Omega)$. The orthogonal projection $P_{N, \Omega}^{1,0}: H_{0}^{1}(\Omega) \rightarrow V_{N}^{0}(\Omega)$ is defined in the usual way. Furthermore, let $N_{b}$ be any positive integer. We define the projection on $\partial \Omega$, as

$$
\begin{aligned}
{ }_{*} \hat{P}_{N_{b}, \partial \Omega}^{1} \hat{v}_{b, \partial \Omega}(\xi, \eta)= & \frac{1}{2}\left((1-\xi) P_{N_{b}, \Lambda_{\eta}}^{1,0} \hat{v}_{b, L_{1}}^{0}(\eta)+(1-\eta) P_{N_{b}, \Lambda_{\xi}}^{1,0} \hat{v}_{b, L_{2}}^{0}(\xi)\right. \\
& \left.+(1+\xi) P_{N_{b}, \Lambda_{\eta}}^{1,0} \hat{v}_{b, L_{3}}^{0}(\eta)+(1+\eta) P_{N_{b}, \Lambda_{\xi}}^{1,0} \hat{v}_{b, L_{4}}^{0}(\xi)\right) \\
& +\frac{1}{4}((1-\xi)(1-\eta) \hat{v}(-1,-1)+(1+\xi)(1-\eta) \hat{v}(1,-1) \\
& +(1+\xi)(1+\eta) \hat{v}(1,1)+(1-\xi)(1+\eta) \hat{v}(-1,1)) .
\end{aligned}
$$

Furthermore,

$$
\begin{aligned}
v_{\Omega}^{0}(x, y)= & \left.\hat{v}_{\Omega}^{0}(\xi, \eta)\right|_{\xi=\xi(x, y), \eta=\eta(x, y)}, \quad v_{b, \partial \Omega}(x, y)=\left.\hat{v}_{b, \partial \Omega}(\xi, \eta)\right|_{\xi=\xi(x, y), \eta=\eta(x, y)}, \\
& { }_{*} P_{N_{b}, \partial \Omega}^{1} v_{b, \partial \Omega}(x, y)=\left.{ }_{*} \hat{P}_{N_{b}, \partial \Omega}^{1} \hat{v}_{b, \partial \Omega}(\xi, \eta)\right|_{\xi=\xi(x, y), \eta=\eta(x, y)} .
\end{aligned}
$$

Then, the first quasi-Legendre orthogonal projection ${ }_{*} P_{N, N_{b}, \Omega}^{1} v$ is defined by

$$
{ }_{*} P_{N, N_{b}, \Omega}^{1} v(x, y)=P_{N, \Omega}^{1,0} v_{\Omega}^{0}(x, y)+{ }_{*} P_{N_{b}, \partial \Omega}^{1} v_{b, \partial \Omega}(x, y) .
$$

It can be checked that ${ }_{*} P_{N, N_{b}, \Omega}^{1} v(x, y)=v(x, y)$ at the four vertices of $\Omega$. Since

$$
{ }_{*} P_{N, N_{b}, \Omega}^{1} v-v=P_{N, \Omega}^{1,0} v_{\Omega}^{0}(x, y)-v_{\Omega}^{0}(x, y)+{ }_{*} P_{N_{b}, \partial \Omega}^{1} v_{b, \partial \Omega}(x, y)-v_{b, \partial \Omega}(x, y),
$$

we have

$$
\left\|\nabla\left({ }_{*} P_{N, N_{b}, \Omega}^{1} v-v\right)\right\|_{\Omega} \leq\left\|\nabla\left(P_{N, \Omega}^{1,0} v_{\Omega}^{0}-v_{\Omega}^{0}\right)\right\|_{\Omega}+\left\|\nabla\left({ }_{*} P_{N_{b}, \partial \Omega}^{1} v_{b, \partial \Omega}-v_{b, \partial \Omega}\right)\right\|_{\Omega} .
$$

For notational convenience, let

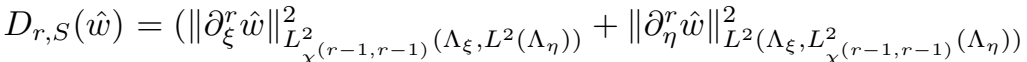

$$
\begin{aligned}
& +\left\|\partial_{\xi}^{r-1} \partial_{\eta} \hat{w}\right\|_{L_{\chi^{(r-2, r-2)}}^{2}\left(\Lambda_{\xi}, L^{2}\left(\Lambda_{\eta}\right)\right)}^{2}+\left\|\partial_{\xi} \partial_{\eta}^{r-1} \hat{w}\right\|_{L^{2}\left(\Lambda_{\xi}, L_{\chi^{(r-2, r-2)}}^{2}\left(\Lambda_{\eta}\right)\right)^{\frac{1}{2}}}^{2} .
\end{aligned}
$$

By (2.5), we have $\|\nabla w\|_{\Omega} \leq c \sigma_{\Omega} \delta_{\Omega}^{-\frac{1}{2}}\left\|\nabla{ }_{S} \hat{w}\right\|_{S}$. Now, let

$$
\phi(x, y)=\left.P_{N, \Lambda_{\xi}}^{1,0} \bullet P_{N, \Lambda_{\eta}}^{1,0} \hat{v}_{\Omega}^{0}\right|_{\xi=\xi(x, y), \eta=\eta(x, y)} .
$$


Then, following the same line as the derivations of (3.32) and (3.33), we deduce that

$$
\begin{aligned}
& \left\|\nabla\left(P_{N, \Omega}^{1,0} v_{\Omega}^{0}-v_{\Omega}^{0}\right)\right\|_{\Omega} \leq\left\|\nabla\left(\phi-v_{\Omega}^{0}\right)\right\|_{\Omega} \leq c \sigma_{\Omega} \delta_{\Omega}^{-\frac{1}{2}}\left\|\nabla_{S}\left(P_{N, \Lambda_{\xi}}^{1,0} \bullet P_{N, \Lambda_{\eta}}^{1,0} \hat{v}_{\Omega}^{0}-\hat{v}_{\Omega}^{0}\right)\right\|_{S} \\
& \leq c \sigma_{\Omega} \delta_{\Omega}^{-\frac{1}{2}} N^{1-r} D_{r, S}\left(\hat{v}_{\Omega}^{0}\right) \leq c \sigma_{\Omega} \delta_{\Omega}^{-\frac{1}{2}} N^{1-r}\left(D_{r, S}(\hat{v})+D_{r, S}\left(\hat{v}_{b, \partial \Omega}^{(1)}\right)+D_{r, S}\left(\hat{v}_{b, \partial \Omega}^{(2)}\right)\right) .
\end{aligned}
$$

We are going to estimate the upper bound of the right side of (7.6). First, an argument similar to the derivation of (3.30), leads to

$$
D_{r, S}(\hat{v}) \leq c \delta_{\Omega}^{-\frac{1}{2}} E_{r, \Omega}(v)
$$

where $E_{r, \Omega}(v)=E_{r, \Omega}^{(1)}(v)+E_{r, \Omega}^{(2)}(v)+E_{r, \Omega}^{(3)}(v)$, with

$$
\begin{aligned}
& E_{r, \Omega}^{(1)}(v)= \sum_{k=0}^{r}\left(\left\|\left(1-\xi^{2}\right)^{\frac{r-1}{2}}\left(a_{1}+a_{3} \eta\right)^{k}\left(b_{1}+b_{3} \eta\right)^{r-k} \partial_{x}^{k} \partial_{y}^{r-k} v\right\|_{\Omega}\right. \\
&\left.\quad+\left\|\left(1-\eta^{2}\right)^{\frac{r-1}{2}}\left(a_{2}+a_{3} \xi\right)^{k}\left(b_{2}+b_{3} \xi\right)^{r-k} \partial_{x}^{k} \partial_{y}^{r-k} v\right\|_{\Omega}\right), \\
& E_{r, \Omega}^{(2)}(v)=\sum_{k=0}^{r-1}\left(\left\|\left(1-\xi^{2}\right)^{\frac{r-2}{2}}\left(a_{1}+a_{3} \eta\right)^{k}\left(b_{1}+b_{3} \eta\right)^{r-k-1}\left(a_{2}+a_{3} \xi\right) \partial_{x}^{k+1} \partial_{y}^{r-k-1} v\right\|_{\Omega}\right. \\
& \quad+\left\|\left(1-\eta^{2}\right)^{\frac{r-2}{2}}\left(a_{2}+a_{3} \xi\right)^{k}\left(b_{2}+b_{3} \xi\right)^{r-k-1}\left(a_{1}+a_{3} \eta\right) \partial_{x}^{k+1} \partial_{y}^{r-k-1} v\right\|_{\Omega} \\
& \quad+\left\|\left(1-\xi^{2}\right)^{\frac{r-2}{2}}\left(a_{1}+a_{3} \eta\right)^{k}\left(b_{1}+b_{3} \eta\right)^{r-k-1}\left(b_{2}+b_{3} \xi\right) \partial_{x}^{k} \partial_{y}^{r-k} v\right\|_{\Omega} \\
&\left.\quad+\left\|\left(1-\eta^{2}\right)^{\frac{r-2}{2}}\left(a_{2}+a_{3} \xi\right)^{k}\left(b_{2}+b_{3} \xi\right)^{r-k-1}\left(b_{1}+b_{3} \eta\right) \partial_{x}^{k} \partial_{y}^{r-k} v\right\|_{\Omega}\right), \\
& E_{r, \Omega}^{(3)}(v)=\sum_{k=0}^{r-2}\left(\left\|\left(1-\xi^{2}\right)^{\frac{r-2}{2}} a_{3}\left(a_{1}+a_{3} \eta\right)^{k}\left(b_{1}+b_{3} \eta\right)^{r-k-2} \partial_{x}^{k+1} \partial_{y}^{r-k-2} v\right\|_{\Omega}\right. \\
& \quad+\left\|\left(1-\eta^{2}\right)^{\frac{r-2}{2}} a_{3}\left(a_{2}+a_{3} \xi\right)^{k}\left(b_{2}+b_{3} \xi\right)^{r-k-2} \partial_{x}^{k+1} \partial_{y}^{r-k-2} v\right\|_{\Omega} \\
& \quad\left\|\left(1-\xi^{2}\right)^{\frac{r-2}{2}} b_{3}\left(a_{1}+a_{3} \eta\right)^{k}\left(b_{1}+b_{3} \eta\right)^{r-k-2} \partial_{x}^{k} \partial_{y}^{r-k-1} v\right\|_{\Omega} \\
&\left.\quad\left\|\left(1-\eta^{2}\right)^{\frac{r-2}{2}} b_{3}\left(a_{2}+a_{3} \xi\right)^{k}\left(b_{2}+b_{3} \xi\right)^{r-k-2} \partial_{x}^{k} \partial_{y}^{r-k-1} v\right\|_{\Omega}\right) .
\end{aligned}
$$

We now estimate $D_{r, S}\left(\hat{v}_{b, \partial \Omega}^{(1)}\right)$. We have from (7.3) that for $r \geq 2$,

$$
\begin{aligned}
\partial_{\xi}^{r} \hat{v}_{b, \partial \Omega}^{(1)}(\xi, \eta) & =\frac{1}{2} \partial_{\xi}^{r}((1-\eta) \hat{v}(\xi,-1)+(1+\eta) \hat{v}(\xi, 1)), \\
\partial_{\eta}^{r} \hat{v}_{b, \partial \Omega}^{(1)}(\xi, \eta) & =\frac{1}{2} \partial_{\eta}^{r}((1-\xi) \hat{v}(-1, \eta)+(1+\xi) \hat{v}(1, \eta)) .
\end{aligned}
$$

On the other hand, for $r \geq 3$,

$$
\begin{aligned}
& \partial_{\xi}^{r-1} \partial_{\eta} \hat{v}_{b, \partial \Omega}^{(1)}(\xi, \eta)=\frac{1}{2} \partial_{\xi}^{r-1}(-\hat{v}(\xi,-1)+\hat{v}(\xi, 1))=\frac{1}{2} \int_{\Lambda_{\eta}} \partial_{\xi}^{r-1} \partial_{\eta} \hat{v}(\xi, \eta) d \eta \\
& \partial_{\xi} \partial_{\eta}^{r-1} \hat{v}_{b, \partial \Omega}^{(1)}(\xi, \eta)=\frac{1}{2} \partial_{\eta}^{r-1}(-\hat{v}(-1, \eta)+\hat{v}(1, \eta))=\frac{1}{2} \int_{\Lambda_{\xi}} \partial_{\xi} \partial_{\eta}^{r-1} \hat{v}(\xi, \eta) d \xi
\end{aligned}
$$

while

$$
\begin{aligned}
\partial_{\xi} \partial_{\eta} \hat{v}_{b, \partial \Omega}^{(1)}(\xi, \eta) & =\frac{1}{2}\left(-\partial_{\eta} \hat{v}(-1, \eta)-\partial_{\xi} \hat{v}(\xi,-1)+\partial_{\eta} \hat{v}(1, \eta)+\partial_{\xi} \hat{v}(\xi, 1)\right) \\
& =\frac{1}{2} \int_{\Lambda_{\eta}} \partial_{\xi} \partial_{\eta} \hat{v}(\xi, \eta) d \eta+\frac{1}{2} \int_{\Lambda_{\xi}} \partial_{\xi} \partial_{\eta} \hat{v}(\xi, \eta) d \xi
\end{aligned}
$$

With the aid of the previous equalities, a direct calculation yields

$$
D_{r, S}\left(\hat{v}_{b, \partial \Omega}^{(1)}\right) \leq c \delta_{\Omega}^{-\frac{1}{2}} F_{r, \Omega}(v)
$$


where $F_{r, \Omega}(v)=F_{r, \Omega}^{(1)}(v)+E_{r, \Omega}^{(2)}(v)+E_{r, \Omega}^{(3)}(v)$, with

$$
\begin{aligned}
F_{r, \Omega}^{(1)}(v)= & \sum_{k=0}^{r}\left(\sum_{\nu=2,4}\left\|\left(1-\xi^{2}\right)^{\frac{r-1}{2}}\left(a_{1}+a_{3} \eta\right)^{k}\left(b_{1}+b_{3} \eta\right)^{r-k} \partial_{x}^{k} \partial_{y}^{r-k} v\right\|_{L^{2}\left(L_{\nu}\right)}\right. \\
& \left.+\sum_{\nu=1,3}\left\|\left(1-\eta^{2}\right)^{\frac{r-1}{2}}\left(a_{2}+a_{3} \xi\right)^{k}\left(b_{2}+b_{3} \xi\right)^{r-k} \partial_{x}^{k} \partial_{y}^{r-k} v\right\|_{L^{2}\left(L_{\nu}\right)}\right) .
\end{aligned}
$$

We next estimate $D_{r, S}\left(\hat{v}_{b, \partial \Omega}^{(2)}\right)$. In fact, $\partial_{\xi}^{r} \hat{v}_{b, \partial \Omega}^{(2)}(\xi, \eta)=\partial_{\eta}^{r} \hat{v}_{b, \partial \Omega}^{(2)}(\xi, \eta)=0$ for $r \geq 2$, and $\partial_{\xi}^{r-1} \partial_{\eta} \hat{v}_{b, \partial \Omega}^{(2)}(\xi, \eta)=\partial_{\xi} \partial_{\eta}^{r-1} \hat{v}_{b, \partial \Omega}^{(2)}(\xi, \eta)=0$ for $r \geq 3$. Moreover,

$$
\begin{aligned}
\partial_{\xi} \partial_{\eta} \hat{v}_{b, \partial \Omega}^{(2)}(\xi, \eta) & =-\frac{1}{4}(\hat{v}(-1,-1)-\hat{v}(1,-1)+\hat{v}(1,1)-\hat{v}(-1,1)) \\
& =-\frac{1}{4} \int_{S} \partial_{\xi} \partial_{\eta} \hat{v}(\xi, \eta) d \xi d \eta
\end{aligned}
$$

As a result, $D_{r, S}\left(\hat{v}_{b, \partial \Omega}^{(2)}\right)=0$ for $r \geq 3$, and

$$
\begin{aligned}
D_{2, S}\left(\hat{v}_{b, \partial \Omega}^{(2)}\right) & \leq\left\|\left(a_{1}+a_{3} \eta\right)\left(a_{2}+a_{3} \xi\right) \partial_{x}^{2} v\right\|_{\Omega}+\left\|\left(b_{1}+b_{3} \eta\right)\left(b_{2}+b_{3} \xi\right) \partial_{y}^{2} v\right\|_{\Omega} \\
& +\left\|\left(a_{1}+a_{3} \eta\right)\left(b_{2}+b_{3} \xi\right) \partial_{x} \partial_{y} v\right\|_{\Omega}+\left\|\left(b_{1}+b_{3} \eta\right)\left(a_{2}+a_{3} \xi\right) \partial_{x} \partial_{y} v\right\|_{\Omega} \\
& +\left\|a_{3} \partial_{x} v\right\|_{\Omega}+\left\|b_{3} \partial_{y} v\right\|_{\Omega} \leq c \delta_{\Omega}^{-\frac{1}{2}}\left(E_{r, \Omega}^{(2)}(v)+E_{r, \Omega}^{(3)}(v)\right) .
\end{aligned}
$$

Furthermore, we estimate $\left\|\nabla\left({ }_{*} P_{N_{b}, \partial \Omega}^{1} v_{b, \partial \Omega}-v_{b, \partial \Omega}\right)\right\|_{\Omega}$. By virtue of (17.3), we have

$$
\begin{aligned}
& * \hat{P}_{N_{b}, \partial \Omega}^{1} \hat{v}_{b, \partial \Omega}-\hat{v}_{b, \partial \Omega}=\frac{1}{2}\left((1-\xi)\left(P_{N_{b}, \Lambda_{\eta}}^{1,0} \hat{v}_{b, L_{1}}^{0}(\eta)-\hat{v}_{b, L_{1}}^{0}(\eta)\right)\right. \\
& \quad+(1-\eta)\left(P_{N_{b}, \Lambda_{\xi}}^{1,0} \hat{v}_{b, L_{2}}^{0}(\xi)-\hat{v}_{b, L_{2}}^{0}(\xi)\right) \\
& \left.\quad+(1+\xi)\left(P_{N_{b}, \Lambda_{\eta}}^{1,0} \hat{v}_{b, L_{3}}^{0}(\eta)-\hat{v}_{b, L_{3}}^{0}(\eta)\right)+(1+\eta)\left(P_{N_{b}, \Lambda_{\xi}}^{1,0} \hat{v}_{b, L_{4}}^{0}(\xi)-\hat{v}_{b, L_{4}}^{0}(\xi)\right)\right) .
\end{aligned}
$$

With the aid of (3.5), we verify that for $r_{b} \geq 2$,

$$
\begin{aligned}
\| \nabla_{S} & \left({ }_{*} \hat{P}_{N_{b}, \partial \Omega}^{1} \hat{v}_{b, \partial \Omega}-\hat{v}_{b, \partial \Omega}\right) \|_{S} \\
& \leq c N_{b}^{1-r_{b}}\left(\left\|\left(1-\eta^{2}\right)^{\frac{r_{b}-1}{2}} \partial_{\eta}^{r_{b}} \hat{v}_{b, L_{1}}^{0}\right\|_{S}+\left\|\left(1-\xi^{2}\right)^{\frac{r_{b}-1}{2}} \partial_{\xi}^{r_{b}} \hat{v}_{b, L_{2}}^{0}\right\|_{S}\right. \\
& \left.+\left\|\left(1-\eta^{2}\right)^{\frac{r_{b}-1}{2}} \partial_{\eta}^{r_{b}} \hat{v}_{b L_{3}}^{0}\right\|_{S}+\left\|\left(1-\xi^{2}\right)^{\frac{r_{b}-1}{2}} \partial_{\xi}^{r_{b}} \hat{v}_{b, L_{4}}^{0}\right\|_{S}\right) \\
& =c N_{b}^{1-r_{b}}\left(\left\|\left(1-\eta^{2}\right)^{\frac{r_{b}-1}{2}} \partial_{\eta}^{r_{b}} \hat{v}(-1, \eta)\right\|_{S}+\left\|\left(1-\xi^{2}\right)^{\frac{r_{b}-1}{2}} \partial_{\xi}^{r_{b}} \hat{v}(\xi,-1)\right\|_{S}\right. \\
& \left.+\left\|\left(1-\eta^{2}\right)^{\frac{r_{b}-1}{2}} \partial_{\eta}^{r_{b}} \hat{v}(1, \eta)\right\|_{S}+\left\|\left(1-\xi^{2}\right)^{\frac{r_{b}-1}{2}} \partial_{\xi}^{r_{b}} \hat{v}(\xi, 1)\right\|_{S}\right) .
\end{aligned}
$$

The above inequality with (2.9) implies

$$
\left\|\nabla\left({ }_{*} P_{N_{b}, \partial \Omega}^{1} v_{b, \partial \Omega}-v_{b, \partial \Omega}\right)\right\|_{\Omega} \leq c \sigma_{\Omega} \delta_{\Omega}^{-1} N_{b}^{1-r_{b}} G_{r_{b}, \Omega}(v)
$$

where

$$
\begin{aligned}
G_{r_{b}, \Omega}(v) & =\sum_{k=0}^{r_{b}}\left(\sum_{\nu=1,3}\left\|\left(1-\eta^{2}\right)^{\frac{r_{b}-1}{2}}\left(a_{2}+a_{3} \xi\right)^{k}\left(b_{2}+b_{3} \xi\right)^{r_{b}-k} \partial_{x}^{k} \partial_{y}^{r_{b}-k} v\right\|_{L^{2}\left(L_{\nu}\right)}\right. \\
& \left.+\sum_{\nu=2.4}\left\|\left(1-\xi^{2}\right)^{\frac{r_{b}-1}{2}}\left(a_{1}+a_{3} \eta\right)^{k}\left(b_{1}+b_{3} \eta\right)^{r_{b}-k} \partial_{x}^{k} \partial_{y}^{r_{b}-k}\right\|_{L^{2}\left(L_{\nu}\right)}\right) .
\end{aligned}
$$

A combination of (7.5)-(7.10) gives

$$
\begin{aligned}
& \left\|\nabla\left({ }_{*} P_{N, N_{b}, \Omega}^{1} v-v\right)\right\|_{\Omega} \\
& \quad \leq c \sigma_{\Omega} \delta_{\Omega}^{-1}\left(N^{1-r}\left(E_{r, \Omega}(v)+F_{r, \Omega}(v)\right)+N_{b}^{1-r_{b}} G_{r_{b}, \Omega}(v)\right) .
\end{aligned}
$$


We now turn to the quasi-Legendre orthogonal approximations corresponding to Neumann boundary conditions imposed on certain edges of $\Omega$. To do this, we need some preparation. For fixedness, we assume that the Neumann boundary condition is given on $L_{1}$; see Figure 1. Let

$$
{ }^{0} H^{1}\left(\Lambda_{\xi}\right)=\left\{v \in H^{1}\left(\Lambda_{\xi}\right) \mid v(1)=0\right\}, \quad{ }^{0} \mathcal{P}_{N}\left(\Lambda_{\xi}\right)=\left\{\phi \in \mathcal{P}_{N}\left(\Lambda_{\xi}\right) \mid \phi(1)=0\right\} .
$$

The orthogonal projection ${ }^{0} P_{N, \Lambda_{\xi}}^{1}:{ }^{0} H^{1}\left(\Lambda_{\xi}\right) \rightarrow{ }^{0} \mathcal{P}_{N}\left(\Lambda_{\xi}\right)$, is defined by

$$
\left(\partial_{\xi}\left({ }^{0} P_{N, \Lambda_{\xi}}^{1} v-v\right), \partial_{\xi} \phi\right)_{\Lambda_{\xi}}=0, \quad \forall \phi \in{ }^{0} \mathcal{P}_{N}\left(\Lambda_{\xi}\right) .
$$

Take

$$
\phi(\xi)=-\int_{\xi}^{1} P_{N-1, \Lambda_{\eta}} \partial_{\eta} v(\eta) d \eta \in{ }^{0} \mathcal{P}_{N}\left(\Lambda_{\xi}\right) .
$$

If $v \in{ }^{0} H\left(\Lambda_{\xi}\right)$ and $\partial_{\xi}^{r} v \in L_{\chi^{(r-1, r-1)}}^{2}\left(\Lambda_{\xi}\right)$ for integers $1 \leq r \leq N+1$, then by virtue of the projection theorem and (3.3), we deduce that

$$
\begin{aligned}
& \left\|\partial_{\xi}\left({ }^{0} P_{N, \Lambda_{\xi}}^{1} v-v\right)\right\|_{\Lambda_{\xi}} \leq\left\|\partial_{\xi}(\phi-v)\right\|_{\Lambda_{\xi}} \\
& \quad=\left\|P_{N-1, \Lambda_{\xi}} \partial_{\xi} v-\partial_{\xi} v\right\|_{\Lambda_{\xi}} \leq c N^{1-r}\left\|\partial_{\xi}^{r} v\right\|_{\chi^{(r-1, r-1)}, \Lambda_{\xi}} .
\end{aligned}
$$

Moreover, a standard duality argument yields

$$
\left\|{ }^{0} P_{N, \Lambda_{\xi}}^{1} v-v\right\|_{\Lambda_{\xi}} \leq c N^{-r}\left\|\partial_{\xi}^{r} v\right\|_{\chi^{(r-1, r-1)}, \Lambda_{\xi}} \cdot
$$

Now, we set

${ }^{0} H^{1}(\Omega)=\left\{v \in H^{1}(\Omega) \mid v=0\right.$ on $L_{2}, L_{3}$ and $\left.L_{4}\right\}, \quad{ }^{0} V_{N}(\Omega)={ }^{0} H^{1}(\Omega) \cap V_{N}(\Omega)$. The orthogonal projection ${ }^{0} P_{N, \Omega}^{1}:{ }^{0} H^{1}(\Omega) \rightarrow{ }^{0} V_{N}(\Omega)$ is defined by

$$
\left(\nabla\left({ }^{0} P_{N, \Omega}^{1} v-v\right), \nabla \phi\right)_{\Omega}=0, \quad \forall \phi \in{ }^{0} V_{N}(\Omega) .
$$

Next, let $\hat{v}_{b, L_{2}}(\xi)=\frac{1}{2} \hat{v}(1,-1)(1+\xi), \hat{v}_{b, L_{4}}(\xi)=\frac{1}{2} \hat{v}(1,1)(1+\xi)$, and let $\hat{v}_{b, L_{3}}(\eta)$ be the same as in (7.1). We also define $\hat{v}_{b, L_{2}}^{0}(\xi), \hat{v}_{b, L_{3}}^{0}(\eta)$ and $\hat{v}_{b, L_{4}}^{0}(\xi)$ by (7.2) with the above new $\hat{v}_{b, L_{2}}(\xi)$ and $\hat{v}_{b, L_{4}}(\xi)$. Evidently, $\hat{v}_{b, L_{2}}^{0}(1)=\hat{v}_{b, L_{3}}^{0}( \pm 1)=\hat{v}_{b, L_{4}}^{0}(1)=0$. Furthermore, we set $\hat{v}_{\Omega}^{0}(\xi, \eta)=\hat{v}(\xi, \eta)-\hat{v}_{b, \partial \Omega}(\xi, \eta)$, with

$$
\begin{aligned}
\hat{v}_{b, \partial \Omega}(\xi, \eta)= & \frac{1}{2}\left((1-\eta) \hat{v}_{b, L_{2}}^{0}(\xi)+(1+\xi) \hat{v}_{b, L_{3}}^{0}(\eta)+(1+\eta) \hat{v}_{b, L_{4}}^{0}(\xi)\right) \\
& +\frac{1}{4}((1+\xi)(1-\eta) \hat{v}(1,-1)+(1+\xi)(1+\eta) \hat{v}(1,1)) .
\end{aligned}
$$

The function $\hat{v}_{\Omega}^{0}(\xi, \eta)$ vanishes on $L_{j}, j=2,3,4$. We also introduce the projection on $\partial \Omega$, as

$$
\begin{aligned}
{ }_{*} \hat{P}_{N_{b}, \partial \Omega}^{1} \hat{v}_{b, \partial \Omega}(\xi, \eta)= & \frac{1}{2}\left((1-\eta)^{0} P_{N_{b}, \Lambda_{\xi}}^{1} \hat{v}_{b, L_{2}}^{0}(\xi)+(1+\xi) P_{N_{b}, \Lambda_{\eta}}^{1,0} \hat{v}_{b, L_{3}}^{0}(\eta)\right. \\
& \left.+(1+\eta)^{0} P_{N_{b}, \Lambda_{\xi}}^{1} \hat{v}_{b, L_{4}}^{0}(\xi)\right) \\
+ & \frac{1}{4}((1+\xi)(1-\eta) \hat{v}(1,-1)+(1+\xi)(1+\eta) \hat{v}(1,1)) .
\end{aligned}
$$

Finally, we define the quasi-Legendre orthogonal projection ${ }_{*} P_{N, N_{b}, \Omega}^{1} v$ as

$$
{ }_{*} P_{N, N_{b}, \Omega}^{1} v(x, y)={ }^{0} P_{N, \Omega}^{1} v_{\Omega}^{0}(x, y)+{ }_{*} P_{N_{b}, \partial \Omega}^{1} v_{b, \partial \Omega}(x, y),
$$

where the meanings of $v_{\Omega}^{0}(x, y), v_{b, \partial \Omega}(x, y)$ and ${ }_{*} P_{N_{b}, \partial \Omega}^{1} v_{b, \partial \Omega}(x, y)$ are similar to those in (7.4), with the new functions $\hat{v}_{\Omega}^{0}(\xi, \eta)$ and $\hat{v}_{b, \partial \Omega}(\xi, \eta)$, and the new projection ${ }_{*} \hat{P}_{N_{b}, \partial \Omega}^{1} \hat{v}_{b, \partial \Omega}(\xi, \eta)$. It can be checked that ${ }_{*} P_{N, N_{b}, \Omega}^{1} v(x, y)=v(x, y)$ at the vertices $(x, y)=(1,-1)$ or $(1,1)$. 
In the same manner, we can define the quasi-Legendre orthogonal projections ${ }_{*} P_{N, N_{b}, \Omega}^{1} v$ corresponding to the Neumann boundary condition imposed on the edges $L_{2}, L_{3}$ and $L_{4}$, respectively. Moreover, by using (3.5), (7.13) and an argument like the derivation of (7.11), we can derive the error estimates for these quasi-Legendre orthogonal projections, which are similar to (7.11).

7.2. Composite quasi-orthogonal approximation on polygons. We are now in a position to study the quasi-Legendre orthogonal projection on a polygon with the boundary $\partial \Omega$ and $\partial^{*} \Omega \subseteq \partial \Omega$. We divide $\Omega$ into convex quadrilaterals $\Omega_{i}, 1 \leq$ $i \leq n$, with the boundary $\partial \Omega_{i}$, the edges $L_{i, \nu}$, the vertices $Q_{i, \nu}$ and the angles $\theta_{i, \nu}, 1 \leq \nu \leq 4$. Therefore, $\partial^{*} \Omega_{i}=\partial^{*} \Omega \cap \partial \Omega_{i}$. The local variable transformation are denoted by $\xi_{i}=\xi_{i}(x, y)$ and $\eta_{i}=\eta_{i}(x, y), 1 \leq i \leq n$. The corresponding quantities $\sigma_{\Omega_{i}}, \gamma_{\Omega_{i}}, \delta_{\Omega_{i}}, d_{i, 1}, d_{i, 2}, a_{i, 1}, a_{i, 2}, a_{i, 3}, b_{i, 1}, b_{i, 2}$ and $b_{i, 3}$ are defined in the same way as for the single quadrilateral; see Section 3 . Let $h_{i}=$ diam $\Omega_{i}$. Assume that the partition of $\Omega$ satisfies the following hypotheses:

$\left(\mathrm{H}_{1}\right) \bar{\Omega}=\bigcup_{i=1}^{n} \bar{\Omega}_{i}$ and $\Omega_{i} \cap \Omega_{k}=\emptyset$ if $i \neq k$,

$\left(\mathrm{H}_{2}\right)$ each vertex of $\Omega_{i}$ is also one of the vertices of adjacent quadrilaterals,

$\left(\mathrm{H}_{3}\right)$ if $\bar{\Omega}_{i} \cap \partial \Omega \neq \emptyset$, then $\Omega_{i}$ has at most one edge $\partial^{*} \Omega_{i}$,

$\left(\mathrm{H}_{4}\right)$ there are positive constants $\beta_{0}$ and $\beta_{1}$ such that $0<\beta_{0} \leq \theta_{i, \nu} \leq \beta_{1}<$ $\pi, 1 \leq \nu \leq 4,1 \leq i \leq n$, and so $0<\delta_{0} h_{i}^{2} \leq \delta_{\Omega_{i}} \leq \delta_{1} h_{i}^{2}, 1 \leq i \leq n$.

Let $N=\left(N_{1}, N_{2}, \cdots, N_{n}\right)$ and $r=\left(r_{1}, r_{2}, \cdots, r_{n}\right)$. We define the quasi-Legendre orthogonal projection ${ }_{*} P_{\mathbf{N}, N_{b}, \Omega}^{1} v$ as $\left.{ }_{*} P_{\mathbf{N}, N_{b}, \Omega}^{1} v\right|_{\Omega_{i}}={ }_{*} P_{N_{i}, N_{b}, \Omega_{i}}^{1} v, 1 \leq i \leq n$, where the projections ${ }_{*} P_{N_{i}, N_{b}, \Omega_{i}}^{1} v$ are constructed in such a way that

(A) if $\bar{\Omega}_{i} \cap \partial \Omega=\emptyset$, then ${ }_{*} P_{N_{i}, N_{b}, \Omega_{i}}^{1} v$ is given by (7.4),

(B) if $\partial^{*} \Omega_{i} \neq \emptyset$, say $\partial^{*} \Omega_{i}=L_{i, 1}$, then ${ }_{*} P_{N_{i}, N_{b}, \Omega_{i}}^{1} v$ is similar to (7.16).

Clearly, if $L_{i, \nu}$ and $L_{k, \nu}$ are the same segment, say $L_{i, 3}=L_{k, 1}$, then the coefficients in the expansions of $P_{N_{b}, \Lambda_{\eta_{i}}}^{1,0} \hat{v}_{b, L_{i, 3}}^{0}\left(\eta_{i}\right)$ and $P_{N_{b}, \Lambda_{\eta_{k}}}^{1,0} \hat{v}_{b, L_{k, 1}}^{0}\left(\eta_{k}\right)$ are the same. It can be checked that ${ }_{*} P_{\mathbf{N}, N_{b}, \Omega}^{1} v \in C(\bar{\Omega})$.

For a description of the approximation error, we introduce the notation $E_{r_{i}, \Omega_{i}}(v)$, $F_{r_{i}, \Omega_{i}}(v)$ and $G_{r_{b}, \Omega_{i}}(v)$, with the quantities $\xi_{i}, \eta_{i}, \sigma_{\Omega_{i}}, \gamma_{\Omega_{i}}, \delta_{\Omega_{i}}, d_{i, 1}, d_{i, 2}, a_{i, 1}, a_{i, 2}$, $a_{i, 3}, b_{i, 1}, b_{i, 2}$ and $b_{i, 3}$, respectively. According to the previous statements and an standard argument as in [2, 5, 7], we observe that if $v \in H^{1}\left(\Omega_{i}\right) \cap C\left(\bar{\Omega}_{i}\right)$ and $E_{r_{i}, \Omega_{i}}(v), F_{r_{i}, \Omega_{i}}(v)$ and $G_{r_{b}, \Omega_{i}}(v)$ are finite for integers $2 \leq r_{i} \leq N_{i}+1(1 \leq i \leq n)$ and $2 \leq r_{b} \leq N_{b}+1$, then

$$
\begin{aligned}
& \left\|\nabla\left({ }_{*} P_{\mathbf{N}, N_{b}, \Omega}^{1} v-v\right)\right\|_{\Omega} \\
& \quad \leq c \sigma_{\Omega} \delta_{\Omega}^{-1} \sum_{i=1}^{n}\left(N_{i}^{1-r_{i}}\left(E_{r_{i}, \Omega_{i}}(v)+F_{r_{i}, \Omega_{i}}(v)\right)+N_{b}^{1-r_{b}} G_{r_{b}, \Omega_{i}}(v)\right) .
\end{aligned}
$$

7.3. Spectral element method for polygons. We now consider the following problem,

$$
\begin{cases}-\Delta U(x, y)=f(x, y), & (x, y) \in \Omega, \\ \partial_{n} U(x, y)=g_{2}(x, y), & (x, y) \in \partial^{*} \Omega, \\ U(x, y)=g_{1}(x, y), & (x, y) \in \partial \Omega \backslash \partial^{*} \Omega .\end{cases}
$$


If $\partial^{*} \Omega=\partial \Omega$, we require, in addition, $(f, 1)_{\Omega}=0$ and $(U, 1)_{\Omega}=0$ for fixing the solution. In what follows, we suppose $\partial^{*} \Omega \neq \partial \Omega$ for simplicity. Let

$$
\begin{aligned}
V_{g_{1}}(\Omega) & =\left\{v \in H^{1}(\Omega) \cap C(\bar{\Omega}) \mid v=g_{1} \text { on } \partial \Omega \backslash \partial^{*} \Omega\right\}, \\
\bar{V}(\Omega) & =\left\{v \in H^{1}(\Omega) \cap C(\bar{\Omega}) \mid v=0 \text { on } \partial \Omega \backslash \partial^{*} \Omega\right\} .
\end{aligned}
$$

The weak formulation of (7.18) is to find $U \in V_{g_{1}}(\Omega)$ such that

$$
(\nabla U, \nabla v)_{\Omega}+\int_{\partial^{*} \Omega} g_{2}(x, y) v(x, y) d s=(f, v)_{\Omega}, \quad \forall v \in \bar{V}(\Omega) .
$$

For the numerical solution of (7.19), we need three kinds of base functions. Let $L_{l}(\xi)$ be the Legendre polynomial of degree $l$ as usual. The base functions corresponding to $\Omega_{i}$, are given by

$$
=\left\{\begin{array}{l}
\psi_{\Omega_{i}, l_{i}, m_{i}}^{0}(x, y) \\
\sqrt{\left(4 l_{i}+6\right)\left(4 m_{i}+6\right)} \\
\left.\left(L_{l_{i}}\left(\xi_{i}\right)-L_{l_{i}+2}\left(\xi_{i}\right)\right)\left(L_{m_{i}}\left(\eta_{i}\right)-L_{m_{i}+2}\left(\eta_{i}\right)\right)\right|_{\xi_{i}=\xi_{i}(x, y), \eta_{i}=\eta_{i}(x, y)}, \quad \text { on } \bar{\Omega}_{i}, \\
0, \quad \text { otherwise. }
\end{array}\right.
$$

Next, we define the base functions corresponding to the edges of quadrilaterals. For instance, if $L_{i, k}=L_{i, 1}=L_{k, 3}$, then the corresponding base function

$$
\begin{aligned}
& \psi_{L_{i, k}, l_{i, k}}^{0}(x, y) \\
& = \begin{cases}\left.\frac{1}{2 \sqrt{4 l_{i, k}+6}}\left(1-\xi_{i}\right)\left(L_{l_{i, k}}\left(\eta_{i}\right)-L_{l_{i, k}+2}\left(\eta_{i}\right)\right)\right|_{\xi_{i}=\xi_{i}(x, y), \eta_{i}=\eta_{i}(x, y)}, & \text { on } \bar{\Omega}_{i}, \\
\left.\frac{1}{2 \sqrt{4 l_{i, k}+6}}\left(1+\xi_{k}\right)\left(L_{l_{i, k}}\left(\eta_{k}\right)-L_{l_{i, k}+2}\left(\eta_{k}\right)\right)\right|_{\xi_{k}=\xi_{k}(x, y), \eta_{k}=\eta_{k}(x, y)} & \text { on } \bar{\Omega}_{k}, \\
0, \text { otherwise, } & \end{cases}
\end{aligned}
$$

The third kind of base functions correspond to the vertices of quadrilaterals. For example, if $Q_{i_{1}, 1}=Q_{i_{2}, 2}=Q_{i_{3}, 3}=Q_{i_{4}, 4}$, then the the corresponding base function

$$
\psi_{Q_{i_{1}, i_{2}, i_{3}, i_{4}}}(x, y)= \begin{cases}\left.\frac{1}{4}\left(1-\xi_{i_{1}}\right)\left(1-\eta_{i_{1}}\right)\right|_{\xi_{i_{1}}=\xi_{i_{1}}(x, y), \eta_{i_{1}}=\eta_{i_{1}}(x, y),} & \text { on } \bar{\Omega}_{i_{1}}, \\ \left.\frac{1}{4}\left(1+\xi_{i_{2}}\right)\left(1-\eta_{i_{2}}\right)\right|_{\xi_{i_{2}}=\xi_{i_{2}}(x, y), \eta_{i_{2}}=\eta_{i_{2}}(x, y),} & \text { on } \bar{\Omega}_{i_{2}}, \\ \left.\frac{1}{4}\left(1+\xi_{i_{3}}\right)\left(1+\eta_{i_{3}}\right)\right|_{\xi_{i_{3}}=\xi_{i_{3}}(x, y), \eta_{i_{3}}=\eta_{i_{3}}(x, y),} & \text { on } \bar{\Omega}_{i_{3}}, \\ \left.\frac{1}{4}\left(1-\xi_{i_{4}}\right)\left(1+\eta_{i_{4}}\right)\right|_{\xi_{i_{4}}=\xi_{i_{4}}(x, y), \eta_{i_{4}}=\eta_{i_{4}}(x, y),} & \text { on } \bar{\Omega}_{i_{4}}, \\ 0, & \text { otherwise. }\end{cases}
$$

Let $W_{\mathbf{N}, N_{b}}(\Omega)$ be the set spanned by $\psi_{\Omega_{i}, l_{i}, m_{i}}^{0}(x, y), 0 \leq l_{i}, m_{i} \leq N_{i}-2$, all $\psi_{L_{i, k}, l_{i, k}}^{0}(x, y), 0 \leq l_{i, k} \leq N_{b}$ and all $\psi_{Q_{i_{1}, i_{2}, i_{3}, i_{4}}}(x, y)$. Clearly, $W_{\mathbf{N}, N_{b}}(\Omega) \subset H^{1}(\Omega) \cap$ $C(\bar{\Omega})$. Furtherore, we let

$$
V_{\mathbf{N}, N_{b}}(\Omega)=\left\{\phi \in W_{\mathbf{N}, N_{b}}(\Omega) \mid \phi=P_{N_{b}, \partial \Omega}^{1} g_{1} \text { on } \partial \Omega \backslash \partial^{*} \Omega\right\},
$$

where, for example, if the edge $L_{i, 1} \subseteq \partial \Omega \backslash \partial^{*} \Omega$, then we take $\hat{g}_{1}\left(-1, \eta_{i}\right)=$ $g_{1}\left(x\left(-1, \eta_{i}\right), y\left(-1, \eta_{i}\right)\right)$ and

$$
\begin{aligned}
{ }_{*} P_{N_{b}, \partial \Omega}^{1} g_{1}(x, y)= & P_{N_{b}, \Lambda_{\eta_{i}}}^{1,0}\left(\hat{g}_{1}\left(-1, \eta_{i}\right)-\frac{1}{2} \hat{g}_{1}(-1,-1)\left(1-\eta_{i}\right)-\frac{1}{2} \hat{g}_{1}(-1,1)\left(1+\eta_{i}\right)\right) \\
& +\frac{1}{2} \hat{g}_{1}(-1,-1)\left(1-\eta_{i}\right)+\left.\frac{1}{2} \hat{g}_{1}(-1,1)\left(1+\eta_{i}\right)\right|_{\xi_{i}=\xi_{i}(x, y), \eta_{i}=\eta_{i}(x, y)} .
\end{aligned}
$$


The set $V_{\mathbf{N}, N_{b}}^{*}(\Omega)=\left\{\phi \in W_{\mathbf{N}, N_{b}}(\Omega) \mid \phi=0\right.$ on $\left.\partial \Omega \backslash \partial^{*} \Omega\right\}$.

We now construct the spectral method for (7.19). We first consider an auxiliary problem (cf. [8]), which is to find the solution $W \in V_{* P_{N_{b}, \partial \Omega}^{1} g_{1}}(\Omega)$ such that

$$
(\nabla W, \nabla v)_{\Omega}+\int_{\partial^{*} \Omega} g_{2}(x, y) v(x, y) d s=(f, v)_{\Omega}, \quad \forall v \in \bar{V}(\Omega) .
$$

The corresponding spectral method is to find $w_{\mathbf{N}, N_{b}} \in V_{\mathbf{N}, N_{b}}(\Omega)$ such that $(7.21)$

$$
\left(\nabla w_{\mathbf{N}, N_{b}}, \nabla \phi\right)_{\Omega}+\int_{\partial^{*} \Omega} g_{2}(x, y) \phi(x, y) d s=(f, \phi)_{\Omega}, \quad \forall \phi \in V_{\mathbf{N}, N_{b}}^{*}(\Omega) .
$$

For derivation of error estimate of numerical solution, we introduce the orthogonal projection $P_{\mathbf{N}, N_{b}, \Omega}^{1} v: H^{1}(\Omega) \cap C(\bar{\Omega}) \rightarrow V_{\mathbf{N}, N_{b}}(\Omega)$ such that

$$
\left(\nabla\left(P_{\mathbf{N}, N_{b}, \Omega}^{1} v-v\right), \nabla \phi\right)_{\Omega}=0, \quad \forall \phi \in V_{\mathbf{N}, N_{b}}^{*}(\Omega)
$$

This, along with (7.20), leads to

$$
\left(\nabla P_{\mathbf{N}, N_{b}, \Omega}^{1} W, \nabla \phi\right)_{\Omega}+\int_{\partial^{*} \Omega} g_{2}(x, y) \phi(x, y) d s=(f, \phi)_{\Omega}, \quad \forall \phi \in V_{\mathbf{N}, N_{b}}^{*}(\Omega) .
$$

Subtracting (7.22) from (7.21) yields

$$
\left(\nabla\left(P_{\mathbf{N}, N_{b}, \Omega}^{1} W-w_{\mathbf{N}, N_{b}}\right), \nabla \phi\right)_{\Omega}=0, \quad \forall \phi \in V_{\mathbf{N}, N_{b}}^{*}(\Omega) .
$$

This implies $w_{\mathbf{N}, N_{b}}=P_{\mathbf{N}, N_{b}, \Omega}^{1} W$. Consequently (cf. [8]),

$$
\begin{aligned}
\left\|\nabla\left(w_{\mathbf{N}, N_{b}}-W\right)\right\|_{\Omega} & =\left\|\nabla\left(P_{\mathbf{N}_{1} N_{b}, \Omega}^{1} W-W\right)\right\|_{\Omega} \leq\left\|\nabla\left({ }_{*} P_{\mathbf{N}, N_{b}, \Omega}^{1} U-W\right)\right\|_{\Omega} \\
& \leq\left\|\nabla\left({ }_{*} P_{\mathbf{N}, N_{b}, \Omega} U-U\right)\right\|_{\Omega}+\|\nabla(U-W)\|_{\Omega} .
\end{aligned}
$$

Furthermore, we have from (7.18) and (7.20) that

$$
\begin{cases}-\Delta(U(x, y)-W(x, y))=0, & (x, y) \in \Omega \\ \partial_{n}(U(x, y)-W(x, y))=0, & (x, y) \in \partial^{*} \Omega \\ U(x, y)-W(x, y)=g_{1}(x, y)-{ }_{*} P_{N_{b}, \partial \Omega}^{1} g_{1}(x, y), & (x, y) \in \partial \Omega \backslash \partial^{*} \Omega\end{cases}
$$

According to the properties of elliptic equation (cf. [8]) and the quasi-Legendre orthogonal approximation, we obtain

$$
\begin{aligned}
\| U- & W \|_{H^{1}(\Omega)} \\
& \leq c\left\|g_{1}-{ }_{*} P_{N_{b}, \partial \Omega}^{1} g_{1}\right\|_{H^{1}\left(\partial \Omega \backslash \partial^{*} \Omega\right)} \leq c \sigma_{\Omega} \delta_{\Omega}^{-1} N_{b}^{1-r_{b}} \sum_{i=1}^{n} K_{r_{b}, \Omega_{i}}\left(g_{1}\right)
\end{aligned}
$$


where $K_{r_{b}, \Omega_{i}}\left(g_{1}\right)=0$ if $\bar{\Omega}_{i} \cap \partial \Omega=\emptyset$, and otherwise,

$$
K_{r_{b}, \Omega_{i}}\left(g_{1}\right)=\left\{\begin{array}{rr}
\sum_{k=0}^{r_{b}}\left\|\left(1-\eta^{2}\right)^{\frac{r_{b}-1}{2}}\left(a_{2}+a_{3} \xi\right)^{k}\left(b_{2}+b_{3} \xi\right)^{r_{b}-k} \partial_{x}^{k} \partial_{y}^{r_{b}-k} g_{1}\right\|_{L^{2}\left(L_{i, 1}\right)}, & \text { if } L_{i, 1} \subseteq \partial \Omega \backslash \partial^{*} \Omega, \\
\sum_{k=0}^{r_{b}}\left\|\left(1-\xi^{2}\right)^{\frac{r_{b}-1}{2}}\left(a_{1}+a_{3} \eta\right)^{k}\left(b_{1}+b_{3} \eta\right)^{r_{b}-k} \partial_{x}^{k} \partial_{y}^{r_{b}-k} g_{1}\right\|_{L^{2}\left(L_{i, 2}\right)}, & \text { if } L_{i, 2} \subseteq \partial \Omega \backslash \partial^{*} \Omega, \\
\sum_{k=0}^{r_{b}}\left\|\left(1-\eta^{2}\right)^{\frac{r_{b}-1}{2}}\left(a_{2}+a_{3} \xi\right)^{k}\left(b_{2}+b_{3} \xi\right)^{r_{b}-k} \partial_{x}^{k} \partial_{y}^{r_{b}-k} g_{1}\right\|_{L^{2}\left(L_{i, 3}\right)}, \\
\text { if } L_{i, 3} \subseteq \partial \Omega \backslash \partial^{*} \Omega, \\
\sum_{k=0}^{r_{b}}\left\|\left(1-\xi^{2}\right)^{\frac{r_{b}-1}{2}}\left(a_{1}+a_{3} \eta\right)^{k}\left(b_{1}+b_{3} \eta\right)^{r_{b}-k} \partial_{x}^{k} \partial_{y}^{r_{b}-k} g_{1}\right\|_{L^{2}\left(L_{i, 4}\right)}, \\
\text { if } L_{i, 4} \subseteq \partial \Omega \backslash \partial^{*} \Omega .
\end{array}\right.
$$

A combination of (7.17), (7.23) and (7.24) leads to the following conclusion.

Theorem 7.1. If the hypotheses $\left(\mathrm{H}_{1}\right)-\left(\mathrm{H}_{4}\right)$ hold, $U \in H^{1}(\Omega) \cap C(\bar{\Omega})$, and all $E_{r_{i}, \Omega_{i}}(U), F_{r_{i}, \Omega_{i}}(U), G_{r_{b}, \Omega_{i}}(U)$ and $K_{r_{b}, \Omega_{i}}\left(g_{1}\right)$ are finite for integers $2 \leq r_{i} \leq$ $N_{i}+1,1 \leq i \leq n$ and $2 \leq r_{b} \leq N_{b}+1$, then

$$
\begin{aligned}
\| U- & w_{\mathbf{N}, N_{b}} \|_{H^{1}(\Omega)} \\
& \leq c \sigma_{\Omega} \delta_{\Omega}^{-1} \sum_{i=1}^{n}\left(N_{i}^{1-r_{i}}\left(E_{r_{i}, \Omega_{i}}(U)+F_{r_{i}, \Omega_{i}}(U)\right)+N_{b}^{1-r_{b}}\left(G_{r_{b}, \Omega_{i}}(U)+K_{r_{b}, \Omega_{i}}\left(g_{1}\right)\right)\right) .
\end{aligned}
$$

Remark 7.1. By (3.26), we have $\max \left(\left|a_{i, 3}\right|,\left|b_{i, 3}\right|,\left|\sigma_{\Omega_{i}}\right|\right) \leq c h_{i}$. Therefore,

$$
\begin{aligned}
\left\|U-W_{\mathbf{N}, N_{b}}\right\|_{H^{1}(\Omega)} & \leq c \sum_{i=1}^{n} N_{i}^{1-r_{i}}\left(\sum_{k=0}^{r_{i}} h_{i}^{r_{i}-1}\left\|\partial_{x}^{k} \partial_{y}^{r_{i}-k} U\right\|_{\Omega}+\sum_{k=0}^{r_{i}-1} h_{i}^{r_{i}-2}\left\|\partial_{x}^{k} \partial_{y}^{r_{i}-k} U\right\|_{\Omega}\right. \\
& \left.+\sum_{\nu=1}^{4} \sum_{k=0}^{r_{i}} h_{i}^{r_{i}-1}\left\|\partial_{x}^{k} \partial_{y}^{r_{i}-k} U\right\|_{L^{2}\left(L_{i, \nu}\right)}\right) \\
& +c \sum_{i=1}^{n} N_{b}^{1-r_{b}} h_{i}^{r_{b}-1} \sum_{\nu=1}^{4} \sum_{k=0}^{r_{b}}\left(\left\|\partial_{x}^{k} \partial_{y}^{r_{b}-k} U\right\|_{L^{2}\left(L_{i, \nu}\right)}\right. \\
& \left.+\left\|\partial_{x}^{k} \partial_{y}^{r_{b}-k} g_{1}\right\|_{L^{2}\left(L_{i, \nu} \cap\left(\partial \Omega-\partial^{*} \Omega\right)\right)}\right) .
\end{aligned}
$$

This is similar to the result (5.4.16) of [5] and the corresponding result of [17] for multidomain pseudospectral method.

Remark 7.2. In actual computation, we evaluate the terms $\int_{\partial * \Omega} g_{2}(x, y) \phi(x, y) d s$ and $(f, \phi)_{\Omega}$ approximately. Thus, in general, there exist two additional errors depending on the accuracy of numerical quadratures and the smoothness of $f$ and $g_{2}$.

\section{Concluding Remarks}

In this paper, we first established the basic results on the orthogonal approximation for arbitrary convex quadrilaterals, which conform the mathematical foundation of the related spectral method possessing the spectral accuracy. Moreover, 
the Jacobi weighted norms appearing in the upper bounds of approximation errors, cover certain singularities of considered functions at the edges or vertices of quadrilaterals.

As examples of applications, the spectral schemes were proposed for two model problems, with the optimal error estimates of numerical solutions. The numerical results demonstrated their high accuracy, and coincide with the analysis well. We also developed a spectral method for convex polygons. Our method could be applied to exterior problems with non-rectangular obstacles.

It is noted that the super-convergence of finite element method was obtained for analytical solutions; see [19. But our results are valid for solutions in certain weighted Sobolev space, which is much more practical.

\section{Appendix A}

We have from (2.3) that

$\left(a_{1} b_{3}-a_{3} b_{1}\right) \xi^{2}-\left(b_{3}\left(x-a_{0}\right)-a_{3}\left(y-b_{0}\right)+a_{2} b_{1}-a_{1} b_{2}\right) \xi-b_{2}\left(x-a_{0}\right)+a_{2}\left(y-b_{0}\right)=0$. If $a_{1} b_{3}-a_{3} b_{1} \neq 0$, then

$$
\begin{aligned}
\xi(x, y) & =\frac{1}{2\left(a_{1} b_{3}-a_{3} b_{1}\right)}\left(b_{3}\left(x-a_{0}\right)-a_{3}\left(y-b_{0}\right)+a_{2} b_{1}-a_{1} b_{2}\right. \\
& \left.+\sigma \sqrt{\left(b_{3}\left(x-a_{0}\right)-a_{3}\left(y-b_{0}\right)+a_{2} b_{1}-a_{1} b_{2}\right)^{2}+4\left(a_{1} b_{3}-a_{3} b_{1}\right)\left(b_{2}\left(x-a_{0}\right)-a_{2}\left(y-b_{0}\right)\right)}\right),
\end{aligned}
$$

where $\sigma=1$, if the points $Q_{j}$ are numbered counterclockwise as in Section 2, and $\sigma=-1$ otherwise. If $a_{1} b_{3}-a_{3} b_{1}=0$, then

$$
\xi(x, y)=-\frac{b_{2}\left(x-a_{0}\right)-a_{2}\left(y-b_{0}\right)}{b_{3}\left(x-a_{0}\right)-a_{3}\left(y-b_{0}\right)+a_{2} b_{1}-a_{1} b_{2}} .
$$

Similarly, we have

$\left(a_{2} b_{3}-a_{3} b_{2}\right) \eta^{2}-\left(b_{3}\left(x-a_{0}\right)-a_{3}\left(y-b_{0}\right)+a_{1} b_{2}-a_{2} b_{1}\right) \eta-b_{1}\left(x-a_{0}\right)+a_{1}\left(y-b_{0}\right)=0$.

If $a_{2} b_{3}-a_{3} b_{2} \neq 0$, then

$$
\begin{aligned}
\eta(x, y) & =\frac{1}{2\left(a_{2} b_{3}-a_{3} b_{2}\right)}\left(b_{3}\left(x-a_{0}\right)-a_{3}\left(y-b_{0}\right)+a_{1} b_{2}-a_{2} b_{1}\right. \\
& \left.+\lambda \sqrt{\left(b_{3}\left(x-a_{0}\right)-a_{3}\left(y-b_{0}\right)+a_{1} b_{2}-a_{2} b_{1}\right)^{2}+4\left(a_{2} b_{3}-a_{3} b_{2}\right)\left(b_{1}\left(x-a_{0}\right)-a_{1}\left(y-b_{0}\right)\right)}\right),
\end{aligned}
$$

where $\lambda=-1$, if the points $Q_{j}$ are numbered clockwise as in Section 2 , and $\lambda=1$ otherwise. If $a_{2} b_{3}-a_{3} b_{2}=0$, then

$$
\eta(x, y)=-\frac{b_{1}\left(x-a_{0}\right)-a_{1}\left(y-b_{0}\right)}{b_{3}\left(x-a_{0}\right)-a_{3}\left(y-b_{0}\right)+a_{1} b_{2}-a_{2} b_{1}} .
$$

\section{REFERENCES}

[1] Bernardi C. and Maday Y., Spectral methods, in Handbook of Numerical Analysis, 209-486, ed. by Ciarlet P. G. and Lions J. L., Elsevier, Amsterdam, 1997. MR,1470226

[2] Bernardi C., Maday Y. and Rapetti F., Discretisations Variationnelles de Problemes aux Limites Elliptique, Collection: Mathematique et Applications, Vol. 45, Springer-Verlag, Berlin, 2004. MR2068204 (2005g:65004)

[3] Boyd J. P., Chebyshev and Fourier Spectral Methods, Second edition, Dover Publication Inc., Mineda, New York, 2001. MR1874071 (2002k:65160)

[4] Canuto C., Hussaini M. Y., Quarteroni A. and Zang T. A., Spectral Methods: Fundamentals in Single Domains, Springer-Verlag, Berlin, 2006. MR2223552 (2007c:65001)

[5] Canuto C., Hussaini M. Y., Quarteroni A. and Zang T. A., Spectral Methods: Evolution to complex Geometries and Applications to Fluid Dynamics, Springer-Verlag, Berlin, 2007. MR2340254 (2009d:76084) 
[6] Funaro D., Polynomial Approximations of Differential Equations, Springer-Verlag, Berlin, 1992. MR.1176949 (94c:65078)

[7] Gottlieb D. and Orszag S. A., Numerical Analysis of Spectral Methods: Theory and Applications, SIAM-CBMS, Philadelphia, 1977. MR0520152 (58:24983)

[8] Guo Benqi, Approximation theory for the $p$-version of the finite element method in three dimensions, Part II: Convergence of the $p$-version of the finite element method, SIAM J. on Numer. Anal. 47 (2009), no. 4, 2578-2611. MR2217381(2007b:65121)

[9] Guo Ben-yu, Spectral Methods and Their Applications, World Scientific, Singapore, 1998. MR1641586 (2000b:65194)

[10] Guo Ben-yu, Jacobi approximations in certain Hilbert spaces and their applications to singular differential equations, J. Math. Anal. Appl., 243 (2000), 373-408. MR.1741531 (2001b:65082)

[11] Guo Ben-yu, Shen Jie and Wang Li-lian, Optical spectral-Galerkin methods using generalized Jacobi polynomials, J. Sci. Comp., 27 (2006), 305-322. MR2285783 (2008f:65233)

[12] Guo Ben-yu and Wang Li-lian, Jacobi approximations in non-uniformly Jacobi-Weighted Sobolev spaces, J. Appr. Theo., 128 (2004), 1-41. MR2063010 (2005h:41010)

[13] Guo Ben-yu and Wang Li-lian, Non-isotropic Jacobi spectral method, Cont. Math., 329 (2003), 157-164. MR2022641 (2004k:65123)

[14] Guo Ben-yu and Wang Li-lian, Error analysis of spectral method on a triangle, Adv. in Comp. Math., 128 (2005), 1-24. MR2291668(2007k:41008)

[15] Guo Ben-yu and Wang Tian-jun, Composite generalized Laguerre-Legendre spectral method with domain decomposition and its application to Fokker-Planck equation in an finite channel, Math. Comp., 78(2009), 129-151. MR2448700

[16] Guo Ben-yu and Wang Tian-jun, Composite Laguerre-Legendre spectral method for exterior problems, Adv. in Comp. Math., DOI: 10.1017/s.10444-008-9112-5.

[17] Karniadakis G. E. and Sherwin S. J., Spectral/hp Element Methods for CFD, The Second Edition, Oxford Univ. Press, Oxford, 2005. MR2165335 (2006j:65001)

[18] Strang G. and Fix G. J., An Analysis of the Finite Element Method, Prentice-Hall, 1973. MR0443377 (56:1747)

[19] Zhang Zhi-ming, Polynomial preserving gradient recovery and a posteriori estimate for bilinear element on irregular quadrilaterals, Int. J. Numer. Anal. Model, 1 (2004), 1-24. MR2052728 (2005b:65119)

Department of Mathematics, Shanghai Normal University, 200234, Shanghai, PeoPLE'S REPUBLIC OF CHINA

Department of Mathematics, Donghua University, 200065, Shanghai, People's RepubLIC OF CHINA 